\title{
Acyclicity of improvements in finite game forms
}

\author{
Nikolai S. Kukushkin*
}

February 24, 2010

\begin{abstract}
Game forms are studied where the acyclicity, in a stronger or weaker sense, of (coalition or individual) improvements is ensured in all derivative games. In every game form generated by an "ordered voting" procedure, individual improvements converge to Nash equilibria if the players restrict themselves to "minimal" strategy changes. A complete description of game forms where all coalition improvement paths lead to strong equilibria is obtained: they are either dictatorial, or voting (or rather lobbing) about two outcomes. The restriction to minimal strategy changes ensures the convergence of coalition improvements to strong equilibria in every game form generated by a "voting by veto" procedure. JEL Classification Number: C 72 .

Key words: Improvement dynamics; Game form; Perfect information; Potential game; Voting by veto
\end{abstract}

\footnotetext{
${ }^{*}$ Russian Academy of Sciences, Dorodnicyn Computing Center, 40, Vavilova, Moscow 119333 Russia E-mail: ququns@inbox.ru
} 


\section{Introduction}

A.-A. Cournot considered best response dynamics long before the expression "game theory" came into use. Such processes were studied in various contexts since then (Topkis, 1979; Moulin, 1984; Vives, 1990; Milgrom and Roberts, 1991; Kandori and Rob, 1995). Monderer and Shapley (1996) started a similar approach to better reply dynamics.

This paper continues the search for natural classes of strategic games where the acyclicity, in a stronger or weaker sense, of (coalition or individual) improvements is ensured (Rosenthal, 1973; Germeier and Vatel', 1974; Sela, 1992; Monderer and Shapley, 1996; Milchtaich, 1996; Holzman and Law-yone, 1997; Konishi et al., 1997; Kukushkin, 1999, 2002ab, 2004, 2007bc; Friedman and Mezzetti, 2001). Unlike most of the previous literature, we only consider game forms, i.e., we put no restrictions on the preferences of the players, only on strategic interactions.

The topic is somewhat related to the study of consistent, or solvable, game forms (Gurvich, 1975, 1988; Moulin, 1976; Peleg, 1978; Abdou 1995, 1998; Abdou and Keiding, 2003; Boros et al., 2007); however, the acyclicity of improvements is a much stronger (and rarer) property than just the existence of an equilibrium.

In the case of two players, a kind of complete description of game forms where all individual improvement paths in all derivative games lead to Nash equilibria was obtained by Boros et al. (2010); for more than two players, there is no clear prospect for that. Weaker notions of acyclicity of individual improvements result in wider classes of game forms, also without clear prospects for a characterization. The most interesting and important class of game forms with acyclic individual improvements is that of games with perfect information (Kukushkin, 2002a); the results of that paper are somewhat extended here. Similar properties of "ordered voting game forms" (Proposition 3.5 and Theorem 3.7) are also established; such game forms have been considered before (e.g., Moulin, 1980, Kukushkin, 1995, or Mariotti, 2000), but improvement dynamics in them seem to have never been studied.

Concerning coalition improvements, a complete description of game forms where all such improvement paths in all derivative games lead to strong equilibria is obtained; not surprisingly, there are not so many of them (Theorem 4.2). A slight weakening of the requirement widens the class significantly. It is shown that "voting by veto" procedures (Mueller, 1978; Peleg, 1978) generate game forms where the convergence of coalition improvements to strong equilibria is ensured if the players restrict themselves to "minimal" strategy changes (Theorem 4.13).

In Section 2 the basic definitions concerning improvement dynamics in finite strategic games are given; the notion of a game form is introduced and examples of game forms ensuring the acyclicity of improvements are provided. Section 3 contains some general results about game forms with acyclic individual improvements; the class of "ordered voting game forms" is defined. Subsection 3.2 is about games with perfect information; Subsection 3.3, about exact potential, a cardinal analog of acyclicity. Section 4 contains the characterization of game forms with acyclic coalition improvements; in Subsection 4.2, voting by veto procedures are defined and their interesting properties proven. 


\section{Basic Notions}

\subsection{Improvement paths in strategic games}

Our basic model is a finite strategic game with ordinal preferences. It is defined by a finite set of players $N$ (we denote $n=\# N$ ), and finite strategy sets $X_{i}$ and ordinal utility functions $u_{i}: X_{N} \rightarrow \mathbb{R}$, where $X_{N}=\prod_{i \in N} X_{i}$, for all $i \in N$. We denote $\mathcal{N}=2^{N} \backslash\{\emptyset\}$ (the set of potential coalitions) and $X_{I}=\prod_{i \in I} X_{i}$ for each $I \in \mathcal{N}$; instead of $X_{N \backslash\{i\}}$ and $X_{N \backslash I}$, we write $X_{-i}$ and $X_{-I}$, respectively. If $n=2$, then $-i$ refers to the partner/rival of player $i$.

Remark. Whenever $v_{i}: \mathbb{R} \rightarrow \mathbb{R}$ is strictly increasing, the functions $u_{i}$ and $v_{i} \circ u_{i}$ represent the same ordering. Therefore, any meaningful definition, condition, statement, etc., involving ordinal utility functions must be invariant to strictly increasing transformations. Auxiliary constructions, however, may well use numeric values.

With every strategic game, a number of improvement relations on $X_{N}$ are associated $\left(i \in N, I \in \mathcal{N}, y_{N}, x_{N} \in X_{N}\right)$ :

$$
\begin{gathered}
y_{N} \triangleright^{\text {Ind }}{ }_{i} x_{N} \rightleftharpoons\left[y_{-i}=x_{-i} \& u_{i}\left(y_{N}\right)>u_{i}\left(x_{N}\right)\right] ; \\
y_{N} \triangleright^{\text {Ind }} x_{N} \rightleftharpoons \exists i \in N\left[y_{N} \triangleright^{\text {Ind }}{ }_{i} x_{N}\right]
\end{gathered}
$$

(individual improvement relation);

$$
\begin{gathered}
y_{N} \triangleright_{I}^{\mathrm{sCo}} x_{N} \rightleftharpoons\left[y_{-I}=x_{-I} \& \forall i \in I\left[u_{i}\left(y_{N}\right)>u_{i}\left(x_{N}\right)\right]\right] ; \\
y_{N} \triangleright^{\mathrm{sCo}} x_{N} \rightleftharpoons \exists I \in \mathcal{N}\left[y_{N} \triangleright^{\mathrm{sCo}}{ }_{I} x_{N}\right]
\end{gathered}
$$

(strong coalition improvement relation);

$$
\begin{gathered}
y_{N} \triangleright_{I}^{\mathrm{wCo}} x_{N} \rightleftharpoons\left[y_{-I}=x_{-I} \& \forall i \in I\left[u_{i}\left(y_{N}\right) \geq u_{i}\left(x_{N}\right)\right] \&\right. \\
\left.\quad \exists i \in I\left[u_{i}\left(y_{N}\right)>u_{i}\left(x_{N}\right)\right]\right] ; \\
y_{N} \triangleright^{\mathrm{wCo}} x_{N} \rightleftharpoons \exists I \in \mathcal{N}\left[y_{N} \triangleright^{\mathrm{wCo}}{ }_{I} x_{N}\right]
\end{gathered}
$$

(weak coalition improvement relation).

Defining the best response correspondence $\mathcal{R}_{i}: X_{-i} \rightarrow 2^{X_{i}}$ for each $i \in N$ in the usual way,

$$
\mathcal{R}_{i}\left(x_{-i}\right)=\underset{x_{i} \in X_{i}}{\operatorname{Argmax}} u_{i}\left(x_{i}, x_{-i}\right)
$$

for every $x_{-i} \in X_{-i}$, we may introduce one more relation:

$$
\begin{gathered}
y_{N} \triangleright^{\mathrm{BR}} x_{i} \rightleftharpoons\left[y_{-i}=x_{-i} \& x_{i} \notin \mathcal{R}_{i}\left(x_{-i}\right) \ni y_{i}\right] ; \\
y_{N} \triangleright^{\mathrm{BR}} x_{N} \rightleftharpoons \exists i \in N\left[y_{N} \triangleright^{\mathrm{BR}}{ }_{i} x_{N}\right]
\end{gathered}
$$

(best response improvement relation). 
It is often convenient to speak of just "an improvement relation" $\triangleright$ without specifying which of the above-defined relations is meant. A maximizer of an improvement relation $\triangleright$, i.e., a strategy profile $x_{N} \in X_{N}$ such that $y_{N} \triangleright x_{N}$ holds for no $y_{N} \in X_{N}$, is an equilibrium: a Nash equilibrium if $\triangleright$ is $\triangleright^{\text {Ind }}$; a ("very") strong equilibrium if $\triangleright$ is $\triangleright^{\text {sCo }}\left(\triangleright^{\text {wCo }}\right.$ ). Every Nash equilibrium is a maximizer of $\triangleright^{\mathrm{BR}}$. If $\mathcal{R}_{i}\left(x_{-i}\right) \neq \emptyset$ for all $i \in N$ and $x_{-i} \in X_{-i}$, then the converse statement is also true; in a finite game, the condition holds automatically.

Following Kukushkin (2004), we consider an arbitrary binary relation $\triangleright$ on a finite set $X$. An improvement path (for $\triangleright$ ) is a (finite or infinite) sequence $\left\{x^{k}\right\}_{k=0,1, \ldots}$ such that $x^{k+1} \triangleright x^{k}$ whenever $k \geq 0$ and $x^{k+1}$ is defined. A finite improvement cycle is an improvement path $x_{N}^{0}, x_{N}^{1}, \ldots, x_{N}^{m}=x_{N}^{0}(m>0)$; a relation is acyclic if it admits no finite improvement cycle. On a finite set, that property is equivalent to the impossibility of an infinite improvement path; therefore, every improvement path, if continued whenever possible, reaches a maximizer (equilibrium) in a finite number of steps.

It is easy to see that a binary relation $\triangleright$ on a finite set $X$ is acyclic if and only if it admits a numeric potential, i.e., a function $P: X \rightarrow \mathbb{R}$ such that

$$
\forall y, x \in X[y \triangleright x \Rightarrow P(y)>P(x)]
$$

the property is also equivalent to the existence of an order potential, i.e., an irreflexive and transitive binary relation $\succ$ on $X$ such that

$$
\forall y, x \in X[y \triangleright x \Rightarrow y \succ x] .
$$

The relation $\triangleright$ is weakly acyclic if every $x \in X$ is connected to a maximizer of $\triangleright$ with an improvement path, i.e., there is a finite improvement path $\left\{x^{0}, \ldots, x^{m}\right\}(m \geq 0)$ such that $x^{0}=x$ and $x^{m}$ is a maximizer. The weak acyclicity does not exclude the possibility that an improvement process may continue indefinitely without reaching an equilibrium; however, this is improbable under reasonable assumptions (Kalai and Schmeidler, 1977; Milchtaich, 1996; Friedman and Mezzetti, 2001).

Clearly, acyclicity implies weak acyclicity, which, in turn, implies the existence of a maximizer of $\triangleright$. Neither statement can be reversed.

An essential feature of the improvement relations in a strategic game defined by (2.1)(2.4) is their disjunctive structure, reflected in (2.1b), etc. It allows us to introduce an intermediate class of properties. We consider an abstract relation $\triangleright$ with a disjunctive structure, i.e., assume that there are a finite set $M$ (in strategic games, $M=N$ for individual improvements and $M=\mathcal{N}$ for coalition improvements) and binary relations $\triangleright_{i}$ on $X$ for each $i \in M$ such that $y \triangleright x \Longleftrightarrow \exists i \in M\left[y \triangleright_{i} x\right]$ for all $y, x \in X$. We say that $\triangleright$ is acyclic under restrictions if there are binary relations $\gg$ and $\gg_{i}$ on $X$ such that, for all $i \in M$ and $y, x \in X$, there holds

$$
\begin{gathered}
y \gg_{i} x \Rightarrow y \triangleright_{i} x \\
\exists y \in X\left[y \triangleright_{i} x\right] \Rightarrow \exists z \in X\left[z \gg_{i} x\right] ; \\
y \bowtie x \Longleftrightarrow \exists i \in M\left[y \gg_{i} x\right]
\end{gathered}
$$


If $\triangleright$ is acyclic, then all conditions (2.6) are satisfied by $\triangleright$ itself as $\gg$, i.e., $\triangleright$ is acyclic under restrictions. In any case, the conditions $(2.6 \mathrm{a})$ and $(2.6 \mathrm{~b})$ imply that every improvement path of $\gg$ is an improvement path of $\triangleright$ and both relations have the same maximizers. It follows immediately that a relation acyclic under restrictions is weakly acyclic.

Restricted acyclicity means that it is possible to impose restrictions on each player's (or coalition's) strategy changes so that whenever an improvement is possible, an admissible improvement is possible as well, and the convergence to an equilibrium is ensured. When an improvement relation is only weakly acyclic, an agreement between the players as to who is allowed to improve at each stage may be needed. (Explicit cooperation can be replaced with a stochastic choice of the player or coalition to move at each step.) The example in Section 7.7 of Kukushkin (2004) clarifies the difference between the weak acyclicity and acyclicity under restrictions.

The (weak or restricted) acyclicity of the individual improvement relation $\triangleright^{\text {Ind }}(2.1)$ in a finite strategic game $\Gamma$ is called the (weak or restricted) finite individual improvement property ((weak or restricted) FIP) of $\Gamma$. Similarly, the (weak or restricted) finite coalition improvement property ((weak or restricted) FCP) refers to the strong coalition improvement relation $\triangleright^{\text {sCo }}$ defined by $(2.2)$; the (weak or restricted) $F C P^{+}$, to the weak coalition improvement relation $\triangleright^{\text {wCo }}$ defined by (2.3); the (weak or restricted) FBRP, to the best response improvement relation $\triangleright^{\mathrm{BR}}$ defined by (2.4). Actually, the FBRP is a restricted FIP. It is easy to see that the following implications hold:

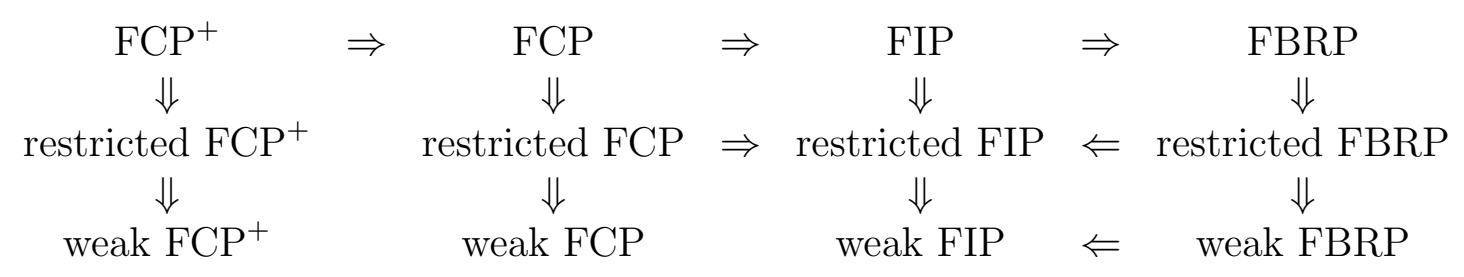

(plus the implication [restricted $\mathrm{FCP}^{+} \Rightarrow$ restricted FIP]).

These properties admit the same dynamic interpretation as the (weak or restricted) acyclicity of an abstract binary relation. The $\mathrm{FCP}\left(^{+}\right)$, FIP, or FBRP ensure that all appropriate adaptive dynamics converge to an equilibrium in a finite number of steps. The weak $\mathrm{FCP}\left({ }^{+}\right)$, weak FIP, or weak FBRP ensure the convergence to an appropriate equilibrium in a finite number of steps with probability one under reasonable assumptions. The properties are also conducive to the convergence of more sophisticated scenarios (Young, 1993; Kandori and Rob, 1995; Friedman and Mezzetti, 2001).

Remark. According to Proposition 6.4 of Kukushkin (2004), weak and restricted FBRP are equivalent for two person games; unfortunately, neither property seems natural for game forms, which are the subject of this paper. The equivalence does not hold w.r.t. the FIP or $\mathrm{FCP}\left({ }^{+}\right)$. 


\subsection{Game forms}

A game form $G$ is defined by a finite set of players $N$, a finite strategy set $X_{i}$ for each $i \in N$, a finite set of outcomes $A$ and a mapping $g: X_{N} \rightarrow A$, where $X_{N}=\prod_{i \in N} X_{i}$ is the set of strategy profiles. For notational simplicity, we assume $X_{i} \cap X_{j}=\emptyset$ whenever $i \neq j$; the assumption is obviously innocuous. We denote $\mathcal{X}=\bigcup_{i \in N} X_{i}$.

Once preferences of the players over the outcomes are specified (and we assume this to be done with ordinal utilities $v_{i}: A \rightarrow \mathbb{R}$ ), a derivative game $G\left(v_{N}\right.$ ) (where $v_{N}$ denotes a list $\left.\left\langle v_{i}\right\rangle_{i \in N}\right)$ emerges, in which the set of players is $N$, the strategy sets are $X_{i}$ 's and utilities are $u_{i}\left(x_{N}\right)=v_{i}\left(g\left(x_{N}\right)\right)$.

If every derivative game $G\left(v_{N}\right)$ possesses a Nash (strong) equilibrium, $G$ is called Nash (strong) consistent. We say that $G$ has the $F I P, F C P$ or $F C P\left({ }^{+}\right)$if so does every derivative game $G\left(v_{N}\right)$. We also use the expression $G$ is an $F I P$, or $F C P\left({ }^{+}\right)$game form. If $G$ has the FIP (FCP), then $G$ is Nash (strongly) consistent; the converse statements are wrong.

Remark. FBRP game forms could be defined quite similarly; however, there is no example of an FBRP game form without the FIP. Moreover, Corollary 2 from Kukushkin (2007a) shows that the FIP and FBRP are equivalent as properties of two person game forms.

Example 2.1. Let us consider four game forms with two players:
a. $\left[\begin{array}{lll}a & a & a \\ b & b & b \\ c & c & c\end{array}\right]$
b. $\left[\begin{array}{lll}a & a & a \\ b & c & c \\ b & d & e\end{array}\right]$
c. $\left[\begin{array}{lll}a & a & a \\ a & b & b \\ a & b & c\end{array}\right]$
d. $\left[\begin{array}{lll}a & a & c \\ a & b & b \\ c & b & c\end{array}\right]$.

It is easily seen that each of them has the FIP, but only the first has FCP (and $\mathrm{FCP}^{+}$at that).

Example 2.2. Let us consider two game forms with two players:
a. $\left[\begin{array}{ccc}a & a & a \\ a & \underline{b} & \underline{c} \\ a & \underline{d} & \underline{e}\end{array}\right]$
b. $\left[\begin{array}{ccc}a & a & a \\ a & \underline{b} & \underline{a} \\ a & \underline{a} & \underline{b}\end{array}\right]$.

It is easily checked that both game forms are Nash consistent; actually, the northwestern corner is a Nash equilibrium for all utilities. Meanwhile, the underlined strategy profiles form an improvement cycle for appropriate utilities in either game form, hence neither has the FIP. However, there is an important difference between them.

Let us consider the first game form with these utility functions: $v_{1}(a)=v_{2}(a)=0$; $v_{1}(c)=v_{1}(d)=v_{2}(b)=v_{2}(e)=1 ; v_{1}(b)=v_{1}(e)=v_{2}(c)=v_{2}(d)=2$. The northwestern corner is a unique Nash equilibrium; an agreement to choose it is self-policing in the usual sense. On the other hand, an agreement not to choose equilibrium strategies is self-policing as well: if I believe that my partner honors the agreement, I have no incentive to cheat (actually, there is no incentive to choose the equilibrium strategy in any case). Moreover, each player would prefer the second agreement, which ensures the choice of one of the underlined outcomes, to the first, notwithstanding the fact that the resulting outcome 
remains unpredictable. The "irrelevance of equilibria" of this kind was discussed by Kreps (1990, pp. 416-417). Example 1.3 in Kukushkin (2002b) demonstrates a similar problem concerning strong equilibria.

One could argue that the second agreement is just to choose a mixed equilibrium; note, however, that our players have ordinal preferences, hence they need not be able to compare probability distributions on the set of outcomes. More technically speaking, a mixed equilibrium whose support consists of the underlined strategy profiles exists for every pair of utility functions representing the same preferences, but its probability distribution is not invariant under monotonic transformations.

No such ugly thing may happen when a game has the weak FIP or weak $\operatorname{FCP}\left(^{+}\right)$ (Theorems 3.1-3.3 of Kukushkin, 2002b). The second game form has the weak FIP, hence is immune to this pathology at least.

Remark. No game form is known such that every derivative game has the weak FIP or weak $\mathrm{FCP}\left({ }^{+}\right)$, but not necessarily a restricted FIP or $\mathrm{FCP}\left(^{+}\right)$.

A fragment $G^{\prime}$ of $G$ is a game form with the same set of players $N$, nonempty subsets $\emptyset \neq X_{i}^{\prime} \subseteq X_{i}$ for all $i \in N$, and the restriction of $g$ to $X_{N}^{\prime}$ as $g^{\prime}$. If $G$ has the FIP (FCP), then so does every fragment of $G$; (strong) Nash consistency, or even a restricted FIP (FCP), need not be "inherited" in this sense.

The notion of restricted acyclicity admits a strengthening when applied to game forms. An admissible change (by coalition $I \in \mathcal{N}$ ) relation is a binary relation $\vdash_{I}$ on $X_{N}$ such that $x_{-I}=y_{-I}$ whenever $x_{N} \vdash_{I} y_{N}$. When $I=\{i\}$, we use the notation $\vdash_{i}$. Such a relation is liberal if

$$
\forall x_{N}, y_{N}\left[\left[y_{-I}=x_{-I} \& g\left(y_{N}\right) \neq g\left(x_{N}\right)\right] \Rightarrow \exists z_{N}\left[x_{N} \vdash_{I} z_{N} \& g\left(z_{N}\right)=g\left(y_{N}\right)\right]\right]
$$

i.e., if every change of outcome available to a coalition (or a player) can be done in an admissible way.

A game form $G$ has an almost unrestricted FIP if there is a list of admissible change relations $\left\langle\vdash_{i}\right\rangle_{i \in N}$ such that every $\vdash_{i}$ is liberal and, in every derivative game $G\left(v_{N}\right)$, the admissible improvement relation $\gg$ defined by (2.6c) with $M=N$ and

$$
y_{N} \gg_{i} x_{N} \rightleftharpoons\left[x_{N} \vdash_{i} y_{N} \& y_{N} \triangleright^{\text {Ind }}{ }_{i} x_{N}\right]
$$

is acyclic. A game form $G$ has an almost unrestricted FCP if there is a list of admissible change relations $\left\langle\vdash_{I}\right\rangle_{I \in \mathcal{N}}$ such that every $\vdash_{I}$ is liberal and, in every derivative game $G\left(v_{N}\right)$, the admissible improvement relation $\gg$ defined by (2.6c) with $M=\mathcal{N}$ and

$$
y_{N} \gg_{I} x_{N} \rightleftharpoons\left[x_{N} \vdash_{I} y_{N} \& y_{N} \triangleright_{I}^{\mathrm{sCo}} x_{N}\right]
$$

is acyclic. Naturally, an almost unrestricted FIP (FCP) of a game form ensures the existence of a (strong) Nash equilibrium in every derivative game, but it is much more than that.

Remark. The game form in Example 2.2b has an almost unrestricted FCP although it seems not to be covered by Theorems 3.7 or 3.14 or 4.13 below. 
In the following theorems, we follow a standardized procedure for the description of admissible changes. We assume that there are costs associated with every change of strategy, i.e., a mapping $\delta_{x_{-I}}: X_{I} \times X_{I} \rightarrow \mathbb{N} \cup\{+\infty\}$ for every $I \in \mathcal{N}$ and $x_{-I} \in X_{-I}$. (When an almost unrestricted FIP is concerned, only singleton $I$ are needed, naturally.) Then a change is admissible if its cost is minimal among all changes leading to the same outcome:

$$
\begin{aligned}
& x_{N} \vdash_{I} y_{N} \rightleftharpoons \\
& \quad\left[y_{-I}=x_{-I} \& \nexists z_{I} \in X_{I}\left[g\left(z_{I}, x_{-I}\right)=g\left(y_{N}\right) \& \delta_{x_{-I}}\left(x_{I}, z_{I}\right)<\delta_{x_{-I}}\left(x_{I}, y_{I}\right)\right]\right] .
\end{aligned}
$$

Since $X_{I}$ is finite, (2.7) holds, i.e., the relation is liberal, for any family of costs $\delta_{x_{-I}}$. In every derivative game $G\left(v_{N}\right)$, an admissible improvement relation $\gg$ is now uniquely defined, based on (2.10), (2.9) or (2.8), and (2.6c) with $M=\mathcal{N}$ or $M=N$.

Clearly, any admissible change relation can be described by (2.10) with appropriate costs: $\delta_{x_{-I}}\left(x_{I}, y_{I}\right)=0$ whenever $\left(x_{I}, x_{-I}\right) \vdash_{I}\left(y_{I}, x_{-I}\right) ; \delta_{x_{-I}}\left(x_{I}, y_{I}\right)=+\infty$ otherwise. The point is that the costs here look reasonable from the viewpoint of intended interpretations. Besides, they can be called "quasidistances" in the sense that they satisfy these requirements: $\delta_{x_{-I}}\left(x_{I}, y_{I}\right)=0 \Longleftrightarrow x_{I}=y_{I}$ and $\delta_{x_{-I}}\left(x_{I}, y_{I}\right) \leq \delta_{x_{-I}}\left(x_{I}, z_{I}\right)+\delta_{x_{-I}}\left(z_{I}, y_{I}\right)$ for all $x_{I}, y_{I}, z_{I} \in X_{I}$ and $x_{-I} \in X_{-I}$ (symmetry holds in some cases, but not always).

\section{FIP Game Forms}

\subsection{Simple and ordered voting game forms}

We start with the definition of a property common for all known FIP game forms. A game form $G$ is separable if it is possible to define a mapping $p: \mathcal{X} \rightarrow A$ such that

$$
g\left(x_{N}\right) \in\left\{p\left(x_{i}\right)\right\}_{i \in N}
$$

for every $x_{N} \in X_{N}$. In principle, the same $g$ may satisfy (3.1) with different mappings $p$; moreover, some $p\left(x_{i}\right)$ may be arbitrary. An interpretation should be clear: choosing a strategy $x_{i}$, player $i$ as if expresses a wish to see $p\left(x_{i}\right)$ chosen; the mapping $g$ determines whose desire will be fulfilled at each strategy profile.

Remark. The present term is due to Vladimir Gurvich (a seminar presentation, 2009). Boros et al. (2010) suggest "assignable game forms"; however, the inevitable association with the "assignment problem" appears undesirable.

Conjecture 3.1. Every FIP game form is separable.

For $n=2$, the statement is proven in Boros et al. (2010, Proposition 9). The converse implication is obviously wrong. Every game form in Examples 2.1 and 2.2 is separable.

The simplest separable game forms are dictatorial ones, where there is a player $i \in N$ such that $g\left(x_{N}\right)=p\left(x_{i}\right)$ for all $x_{N} \in X_{N}$; see Example 2.1a. Unless $g\left(X_{N}\right)$ is a singleton, there cannot be more than one dictator; $p\left(x_{j}\right)$ for $j \neq i$ may be arbitrary. 
A game form is simple if it is separable and there is a linear order on $\mathcal{X}$ such that

$$
g\left(x_{N}\right)=p\left(\min _{i \in N} x_{i}\right)
$$

for every $x_{N} \in X_{N}$. (Again, the same $g$ may be described by (3.2) with different orders on $\mathcal{X}$.) Besides a desirable outcome, every strategy specifies a priority of the desire; then the choice with the highest priority is implemented. If $G$ is dictatorial, we may define $x_{i}<x_{j}$ for every strategy of the dictator $i$ and all $j \neq i$. Every fragment of a simple game form is simple as well.

Remark. Boros et al. (2010) call such game forms "totally reducible"; however, a lone adjective seems preferable.

Given a game form, we call $x_{i} \in X_{i}$ simple if $\# g\left(x_{i}, X_{-i}\right)=1$.

Proposition 3.2. A game form $G$ is simple if and only if there is a simple strategy in every fragment of $G$.

Proof. If $G$ is simple and $G^{\prime}$ is a fragment of $G$, we pick the minimal strategy in $\bigcup_{i \in N} X_{i}^{\prime}$; (3.2) immediately implies that it is simple in $G^{\prime}$. Conversely, we pick a simple strategy in $\mathcal{X}$ and declare it the least in $\mathcal{X}$; then we forget it and define a linear order on $\mathcal{X}$ by induction.

Theorem 3.3. Every simple game form has the FIP.

Proof. Suppose to the contrary that $x_{N}^{0}, x_{N}^{1}, \ldots, x_{N}^{m}=x_{N}^{0}(m>0)$ is an individual improvement cycle in a derivative game $G\left(v_{N}\right)$. Without restricting generality, we may assume that there is no shorter improvement cycle. For each $i \in N$, we define $X_{i}^{\prime}=\left\{x_{i}^{0}, x_{i}^{1}, \ldots, x_{i}^{m-1}\right\}$. By Proposition 3.2, the fragment contains a simple strategy; let it be $x_{i}^{k}$. Without restricting generality, we may assume that $x_{N}^{k} \triangleright^{\text {Ind }}{ }_{i} x_{N}^{k-1}$, hence $x_{N}^{k+1} \triangleright^{\text {Ind }}{ }_{j} x_{N}^{k}$ with $j \neq i$. Since $x_{i}^{k}=x_{i}^{k+1}$ and $x_{i}^{k}$ is simple, we have $g\left(x_{N}^{k}\right)=g\left(x_{N}^{k+1}\right)$, which contradicts the supposed inequality $u_{j}\left(x_{N}^{k+1}\right)>u_{j}\left(x_{N}^{k}\right)$.

All game forms in Example 2.1 except the last one are simple. Theorem 6 of Boros et al. (2010) implies that every two person game form which has the FIP but is not simple must contain that matrix. When $n>2$, even that much cannot be asserted, see Example 4.11 below.

Proposition 3.4. Every $2 \times 2$ fragment of a FIP game form contains a simple strategy, i.e., is simple itself.

Proof. Every fragment of a FIP game form has the FIP, hence is Nash consistent. Now the necessary condition from Gurvich (1975) and Moulin (1976) applies.

Remark. The converse implication holds only for $n=2$ (Kukushkin, 2007a). 
"Dually" to dictatorial game forms, priorities of the strategies may be determined by the outcomes pointed to. Let $A$ be linearly ordered and

$$
g\left(x_{N}\right)=\min _{i \in N} p\left(x_{i}\right)
$$

for each $x_{N} \in X_{N}$; Example 2.1c presents such a game form. A possible interpretation: each player chooses a maximal "level of cooperation" she is ready to accept; then the highest level acceptable to everybody becomes the norm. The term unanimity game forms may be appropriate.

Proposition 3.5. Every unanimity game form has the FIP.

Proof. The game form is simple: $y_{i}>x_{j}$ whenever $p\left(y_{i}\right)>p\left(x_{j}\right)$; the strategies with the same $p\left(x_{i}\right)$ are ordered arbitrarily. Now Theorem 3.3 applies.

Generalizing the notion, we may assume that an outcome is socially acceptable if a certain fraction of players finds it so; the median is most usual in political sciences. Moulin (1980) considered such game forms, with the addition of "fixed votes" to the players' choices, and established some nice properties of them when the preferences are single-peaked. Here we abandon the anonymity requirement and allow arbitrary preferences. Thus, the order on the set of outcomes becomes an element of the decision making procedure, unrelated to the players' preferences.

An ordered voting game form is defined by the following construction. $A$ is linearly ordered and $X_{i}=A$ for each $i \in N$. Strictly speaking, our assumption $X_{i} \cap X_{j}=\emptyset$ is thus violated, but it does not matter here. For each $i \in N$, a "weight" $\mu_{i} \geq 0$ is given; for each $a \in A$, a number $\lambda_{a}>0$. We assume that $\lambda_{a}$ decreases in $a$. Given $x_{N} \in X_{N}$ and $a \in A$, we denote $N^{-}\left(a, x_{N}\right)=\left\{i \in N \mid x_{i}<a\right\}, \varkappa^{-}\left(a, x_{N}\right)=\sum_{i \in N^{-}\left(a, x_{N}\right)} \mu_{i}$, and define

$$
g\left(x_{N}\right)=\max \left\{a \in A \mid \varkappa^{-}\left(a, x_{N}\right)<\lambda_{a}\right\} .
$$

The interpretation is that each player chooses a "personal cap" $x_{i}$ supported by her weight $\mu_{i} ; \lambda_{a}$ is the minimal total weight that makes $a$ ineligible when put against it.

Remark. Since $N$ and $A$ are finite, it seems plausible that integer $\mu$ 's and $\lambda$ 's are sufficient to generate all ordered voting game forms; however, I have not studied the question carefully.

If all $\mu_{i}=1$ and $\lambda_{a}=1$, we have a unanimity game form, the FIP of which was established by Proposition 3.5; if all $\mu_{i}=1$ and $\lambda_{a}=n$, we have $g\left(x_{N}\right)=\max _{i \in N} x_{i}$, i.e., Proposition 3.5 is still applicable after the order on $A$ is reversed. Generally, there is no FIP.

Example 3.6. Let us consider an ordered voting game form $G$ with $N=\{1,2\}, A=$ $\{a, b, c\}(a>b>c)$, both $\mu_{i}=1, \lambda_{c}=\lambda_{b}=2$, and $\lambda_{a}=1$ (median voting scheme with a fixed vote at $b$ ). The game form is described by the following matrix:

$$
\begin{array}{ccc}
\underline{a} & b & \underline{b} \\
b & b & b \\
\underline{b} & b & \underline{c}
\end{array}
$$


The underlined outcomes form an improvement cycle in $G\left(v_{N}\right)$ with appropriate utilities; therefore, $G$ does not have the FIP (although it is Nash consistent). Somewhat unnatural behavior may be observed in the cycle: in the "clockwise" movement, player 2 switches from $a$ to $b$ by choosing $c$, and from $c$ to $b$ by choosing $a$.

Given $a, b \in A$, we define the order distance between them as

$$
d(a, b)=\#\{x \in A \mid \min \{a, b\} \leq x<\max \{a, b\}\}
$$

then we define an admissible change relation by (2.10) with $d$ as $\delta_{x_{-i}}$ for every $i \in N$ and $x_{-i} \in X_{-i}$. In other words, a change of strategy is admissible if the same change of outcome could not be produced by a shorter movement of $x_{i}$.

Theorem 3.7. Every ordered voting game form has an almost unrestricted FIP with the "costs" on each $X_{i}$ defined by (3.5) [independently of $x_{-i}$ ].

Proof. We denote $M=\left\{\mu_{i}\right\}_{i \in N} \subset \mathbb{R}$ and $N(m)=\left\{i \in N \mid \mu_{i} \geq m\right\}$ for each $m \in M$. Given $x_{N} \in X_{N}$ and $m \in M$, we define $\gamma_{m}^{-}\left(x_{N}\right)=\max \left\{a \in A \mid \varkappa^{-}\left(a, x_{N}\right)+m<\lambda_{a}\right\}$ and $\gamma_{m}^{+}\left(x_{N}\right)=\max \left\{a \in A \mid \varkappa^{-}\left(a, x_{N}\right)-m<\lambda_{a}\right\}$; clearly, $\gamma_{m}^{-}\left(x_{N}\right) \leq g\left(x_{N}\right) \leq \gamma_{m}^{+}\left(x_{N}\right)$. Then we define $C_{m}\left(x_{N}\right)=\left\{a \in A \mid \gamma_{m}^{-}\left(x_{N}\right) \leq a \leq \gamma_{m}^{+}\left(x_{N}\right)\right\}, B_{m}\left(x_{N}\right)=\left\{i \in N(m) \mid x_{i} \in C_{m}\left(x_{N}\right)\right\}$, $P_{m}\left(x_{N}\right)=\sum_{i \in B_{m}\left(x_{N}\right)} v_{i}\left(x_{i}\right)$, and a family of lexicographic binary relations on $X_{N}$ :

$$
\begin{aligned}
y_{N} \succeq_{m} x_{N} \rightleftharpoons & {\left[C _ { m } ( y _ { N } ) \subset C _ { m } ( x _ { N } ) \text { or } \left[C_{m}\left(y_{N}\right)=C_{m}\left(x_{N}\right) \&\right.\right.} \\
& \left.\left.\left(B_{m}\left(y_{N}\right) \supset B_{m}\left(x_{N}\right) \text { or }\left[B_{m}\left(y_{N}\right)=B_{m}\left(x_{N}\right) \& P_{m}\left(y_{N}\right) \geq P_{m}\left(x_{N}\right)\right]\right)\right]\right] ; \\
y_{N} \succ_{m} x_{N} \rightleftharpoons & {\left[\begin{array}{c}
C_{m}\left(y_{N}\right) \subset C_{m}\left(x_{N}\right) \text { or }\left[C_{m}\left(y_{N}\right)=C_{m}\left(x_{N}\right) \&\right. \\
\left.\left.\left(B_{m}\left(y_{N}\right) \supset B_{m}\left(x_{N}\right) \text { or }\left[B_{m}\left(y_{N}\right)=B_{m}\left(x_{N}\right) \& P_{m}\left(y_{N}\right)>P_{m}\left(x_{N}\right)\right]\right)\right]\right] .
\end{array}\right.}
\end{aligned}
$$

Clearly,

$$
y_{N} \succ_{m} x_{N} \Longleftrightarrow\left[y_{N} \succeq_{m} x_{N} \& x_{N} \succeq_{m} y_{N}\right]
$$

Finally, a lexicographic aggregate is formed of $\succeq_{m}(m \in M)$ :

$$
y_{N} \succ x_{N} \rightleftharpoons \exists m \in M\left[y_{N} \succ_{m} x_{N} \& \forall m^{\prime}>m\left[y_{N} \succeq_{m^{\prime}} x_{N}\right]\right] .
$$

Obviously, $\succ$ is a strict order; we'll show that it is a potential, in the sense of (2.5b), for admissible improvements. Let $i \in N$ and $x_{N}, y_{N} \in X_{N}$ be such that $y_{-i}=x_{-i}$; we denote $a=g\left(x_{N}\right)$ and $b=g\left(y_{N}\right)$, and assume $b \neq a$. For every $c \in A$ (except $c=\max A$ ), we denote $c+1$ the next point in $A$, uniquely defined by $c+1>c$ and $d(c, c+1)=1$.

Step 3.7.1. $b \in C_{\mu_{i}}\left(x_{N}\right)$.

Proof. Since $\varkappa^{-}\left(b, y_{N}\right)<\lambda_{b}$ while $\varkappa^{-}\left(b, y_{N}\right) \geq \varkappa^{-}\left(b, x_{N}\right)-\mu_{i}$, we have $\varkappa^{-}\left(b, x_{N}\right)<\lambda_{b}+\mu_{i}$, hence $b \geq \gamma_{\mu_{i}}^{-}\left(x_{N}\right)$. For each $c>\gamma_{\mu_{i}}^{+}\left(x_{N}\right)$, we have $\varkappa^{-}\left(c, y_{N}\right) \geq \varkappa^{-}\left(c, x_{N}\right)-\mu_{i} \geq \lambda_{c}$, hence $c \neq b$; therefore, $b \leq \gamma_{\mu_{i}}^{+}\left(x_{N}\right)$. 
Step 3.7.2. $x_{N} \vdash_{i} y_{N} \Longleftrightarrow y_{i}=b$.

Proof. If $x_{i}<a$, then $\varkappa^{-}\left(a, y_{N}\right) \leq \varkappa^{-}\left(a, x_{N}\right)<\lambda_{a}$, hence $b>a$. If $x_{i}>a$, then $\varkappa^{-}(a+$ $\left.1, y_{N}\right) \geq \varkappa^{-}\left(a+1, x_{N}\right) \geq \lambda_{a+1}$, hence $b<a$.

Let $b>a$; then $x_{i} \leq a$. If $y_{i}<b$, then $\varkappa^{-}\left(b, y_{N}\right)=\varkappa^{-}\left(b, x_{N}\right) \geq \lambda_{b}$ : a contradiction. Obviously $\min _{y_{i} \geq b} d\left(x_{i}, y_{i}\right)$ is attained when $y_{i}=b$.

Let $b<a$; then $x_{i} \geq a$. If $y_{i}>b$, then $\varkappa^{-}\left(b+1, y_{N}\right)=\varkappa^{-}\left(b+1, x_{N}\right)<\lambda_{b+1}$ : a contradiction. Obviously $\min _{y_{i} \leq b} d\left(x_{i}, y_{i}\right)$ is attained when $y_{i}=b$.

In the following, we assume $y_{i}=b$.

Step 3.7.3. $C_{m}\left(y_{N}\right) \subseteq C_{m}\left(x_{N}\right)$ for each $m \geq \mu_{i}$.

Proof. Let $b>a$; then $\varkappa^{-}\left(c, y_{N}\right) \leq \varkappa^{-}\left(c, x_{N}\right)$ for each $c \leq b$, while $\varkappa^{-}\left(c, y_{N}\right)=\varkappa^{-}\left(c, x_{N}\right)$ for each $c>b$. Since $\gamma_{m}^{-}\left(x_{N}\right) \leq a<b \leq \gamma_{m}^{+}\left(x_{N}\right)$, we have $\gamma_{m}^{-}\left(y_{N}\right) \geq \gamma_{m}^{-}\left(x_{N}\right)$ and $\gamma_{m}^{+}\left(y_{N}\right)=$ $\gamma_{m}^{+}\left(x_{N}\right)$.

The case of $b<a$ is treated dually.

Step 3.7.4. $y_{N} \succeq_{m} x_{N}$ for each $m>\mu_{i}$.

Proof. Indeed, $C_{m}\left(y_{N}\right) \subseteq C_{m}\left(x_{N}\right)$ by Step 3.7.3. If the inclusion is strict, we even have $y_{N} \succ_{m} x_{N}$. Otherwise, $B_{m}\left(y_{N}\right)=B_{m}\left(x_{N}\right)$ and $P_{m}\left(y_{N}\right)=P_{m}\left(x_{N}\right)$ since $y_{N(m)}=x_{N(m)}$.

Step 3.7.5. If $v_{i}(b)>v_{i}(a)$, then $y_{N} \succ_{\mu_{i}} x_{N}$.

Proof. Again, $C_{\mu_{i}}\left(y_{N}\right) \subseteq C_{\mu_{i}}\left(x_{N}\right)$ by Step 3.7.3. Since $i \in B_{\mu_{i}}\left(y_{N}\right)$, we only have to consider the case of $C_{\mu_{i}}\left(y_{N}\right)=C_{\mu_{i}}\left(x_{N}\right)$ and $B_{\mu_{i}}\left(y_{N}\right)=B_{\mu_{i}}\left(x_{N}\right)$, i.e., $i \in B_{\mu_{i}}\left(x_{N}\right)$. If $x_{i}=a$, we have $P_{\mu_{i}}\left(y_{N}\right)-P_{\mu_{i}}\left(x_{N}\right)=v_{i}(b)-v_{i}(a)>0$. Suppose $x_{i}<a$; then $\gamma_{\mu_{i}}^{-}\left(x_{N}\right) \leq x_{i}<a$ and $b=y_{i}>a$. On the other hand, $\varkappa^{-}\left(a, y_{N}\right)=\varkappa^{-}\left(a, x_{N}\right)-\mu_{i}$, hence $\varkappa^{-}\left(a, y_{N}\right)+\mu_{i}<\lambda_{a}$, hence $\gamma_{\mu_{i}}^{-}\left(y_{N}\right) \geq a>\gamma_{\mu_{i}}^{-}\left(x_{N}\right)$, hence $C_{\mu_{i}}\left(y_{N}\right) \subset C_{\mu_{i}}\left(x_{N}\right)$, contradicting our assumption. The case of $x_{i}>a$ is treated dually.

In the light of Steps 3.7.4 and 3.7.5, the proof of the theorem is accomplished.

Theorem 3.7 becomes wrong if strategy sets $X_{i} \subset A$ are allowed: the assumption $X_{2}=$ $\{a, c\}$ in Example 3.6 would make the changes made by player 2 along the cycle unique.

Remark. In the absence of fixed votes, i.e., when $\lambda_{a}$ is the same for all $a \in A$, an ordered voting game form is obviously separable: $p\left(x_{i}\right)=x_{i}$. The statement seems to be wrong generally although I have not studied the question carefully. 


\subsection{Games with perfect information}

The most important examples of game forms with FIP or almost unrestricted FIP are provided by the normal form of games with perfect information. Following Kukushkin (2002a), we reproduce the familiar concepts in a fashion most convenient for our purposes.

A perfect information game form $(P I G F)$ is a game form with arbitrary (finite) sets $N$ and $A$, and strategies and the mapping $g$ generated by a construction as follows. A game tree $K$ is a finite partially ordered set satisfying these two conditions. (a) For every $\alpha \in K$, the set $\{\beta \in K \mid \beta \leq \alpha\}$ is a chain. (b) There exists the minimum $\alpha_{0}$ of $K$ (the origin), $\alpha_{0} \leq \alpha$ for every $\alpha \in K$. The existence of the meet (greatest common lower bound) $\alpha \wedge \beta$ for every $\alpha, \beta \in K$ easily follows. We call $\beta \in K$ an immediate successor of $\alpha \in K$ if $\alpha<\beta$ while $\alpha<\beta^{\prime}<\beta$ is impossible; the set of all immediate successors of $\alpha \in K$ is denoted $X_{\alpha}$. Imagining an arc from every $\alpha \in K$ to every $\beta \in X_{\alpha}$ turns $K$ into a tree in a geometrical sense.

The set of maximizers of the order on $K$ is denoted $T$ (terminal nodes); the set $K \backslash T, D$ (decision nodes). There is an ownership mapping $\nu: D \rightarrow N$; player $i$ moves at nodes from $D_{i}=\nu^{-1}(i)$. We denote $X_{i}=\prod_{\alpha \in D_{i}} X_{\alpha}$ for $i \in N$ and $X_{N}=\prod_{i \in N} X_{i}$. We identify $X_{N}$ with $\prod_{\alpha \in D} X_{\alpha}$. For every $x_{N} \in X_{N}$ and $\alpha \in D$, we denote $\pi\left(\alpha, x_{N}\right) \subseteq K$ the intersection of all subsets $K^{\prime}$ of $K$ satisfying these two conditions: $\alpha \in K^{\prime}$ and $\left[\beta \in K^{\prime} \Rightarrow x_{\beta} \in K^{\prime}\right.$;; note that $\pi\left(\alpha, x_{N}\right)$ is a chain: a play of the game starting at $\alpha$. The unique element of $T \cap \pi\left(\alpha, x_{N}\right)$ is denoted $\tau\left(\alpha, x_{N}\right)$ : the result of playing $x_{N}$ starting at $\alpha$.

Finally, there is a mapping $\gamma: T \rightarrow A$ and $g\left(x_{N}\right)=\gamma\left(\tau\left(\alpha_{0}, x_{N}\right)\right)$. If $\gamma$ is a bijection, $G$ is called free; in this case, we may just assume $A=T$ (as was done in Kukushkin, 2002a). For each $\alpha \in D$, we denote $F(\alpha)=\gamma(\{\beta \in T \mid \beta>\alpha\})$, the set of outcomes feasible if the play passes through $\alpha$.

To avoid pathologies, we always assume that $\# X_{\alpha}>1$ and $\# F(\alpha)>1$ for every $\alpha \in D$ (i.e., there is no "meaningless" decision node), while $X_{\alpha} \cap D_{i}=\emptyset$ for all $i \in N$ and $\alpha \in D_{i}$ (i.e., no player can make two consecutive choices). The restrictions are innocuous and allow more compact formulations of Theorem 3.9, Conjecture 3.11, and Proposition 3.20 below.

Proposition 3.8. A game form is simple if and only if it can be represented as a perfect information game form where $D$ is a chain in the tree order, i.e., there exists a play of the game containing all decision nodes (one of the above restrictions may have to be violated, namely, $\# F(\alpha)=1$ for some $\alpha \in D$ is possible).

Proof. Straightforward.

Theorem 3.9 (Theorem 1 of Kukushkin, 2002a). If $G$ is a free perfect information game form, then $G$ has the FIP if and only if each $D_{i}$ is a chain in the tree order, i.e., for each player there exists a play of the game containing all his decision nodes.

The sufficiency part, naturally, holds for every PIGF, which cannot be said about the necessity. 
Example 3.10. Let us consider a three-person perfect information game form with five decision nodes, six terminal nodes, and five outcomes. We assume $D_{1}=\left\{\alpha_{0}, \alpha_{2}, \alpha_{3}\right\}$, $D_{2}=\left\{\beta_{2}\right\}, D_{3}=\left\{\beta_{3}\right\}$ and $A=\{a, b, c, d, e\} ; \gamma$ is described by putting outcomes at terminal nodes.

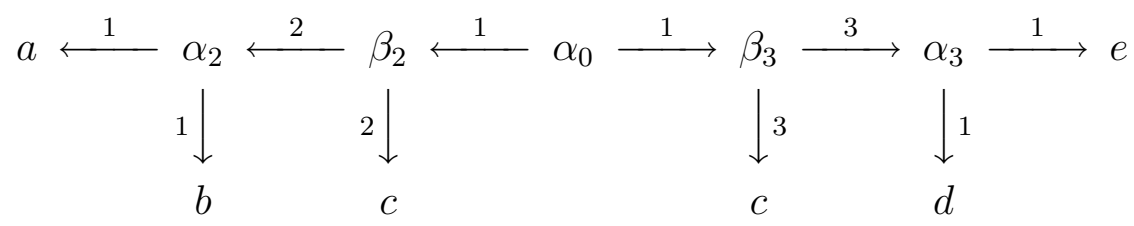

The FIP is not difficult to check.

In an attempt to extend Theorem 3.9 to the general case, we introduce the following requirements. First, the decision nodes of each player form a subsemilattice of the game tree, i.e., if a player moves at two incomparable nodes, he must move at their meet too:

$$
\alpha, \alpha^{\prime} \in D_{i} \Rightarrow \alpha \wedge \alpha^{\prime} \in D_{i}
$$

The second condition needs some auxiliary notations. For $i \in N$ and $\alpha, \beta \in D_{i}$, we denote:

$$
\begin{gathered}
F^{*}(\alpha, \beta)=\gamma\left(\left\{t \in T \mid \alpha<t \wedge \beta<\beta \& t \wedge \beta \notin D_{i}\right\}\right) ; \\
F^{* *}(\alpha, \beta)= \begin{cases}F^{*}(\alpha, \beta), & \text { if } \# F(\beta)>2 ; \\
F^{*}(\alpha, \beta) \backslash F(\beta), & \text { if } \# F(\beta)=2 .\end{cases}
\end{gathered}
$$

Now the condition is

$$
\begin{aligned}
& \forall i \in N \forall \alpha \in D_{i} \forall \beta^{\prime}, \beta^{\prime \prime} \in D_{i} \backslash\{\alpha\} \forall c^{\prime}, c^{\prime \prime} \in A \\
& \quad\left[\beta^{\prime} \wedge \beta^{\prime \prime}=\alpha \& c^{\prime} \in F^{* *}\left(\alpha, \beta^{\prime}\right) \& c^{\prime \prime} \in F^{* *}\left(\alpha, \beta^{\prime \prime}\right) \Rightarrow c^{\prime}=c^{\prime \prime}\right] .
\end{aligned}
$$

If $D_{i}$ is a chain, then (3.6a) holds trivially while (3.6b) holds by default. Generally, conditions (3.6) mean that whenever a player makes the play to turn towards one of her decision nodes rather than towards another, and then another player intervenes denying the first player any further participation in the play, the outcome is "almost" predetermined.

Conjecture 3.11. A perfect information game form has the FIP if and only if it satisfies assumptions (3.6).

Actually, assumptions (3.6) are equivalent to the impossibility of improvement cycles as in Examples 3 and 4 of Kukushkin (2002a), so their necessity is straightforward.

Proposition 3.12. Every perfect information game form satisfying assumptions (3.6) is separable.

Proof. Given $i \in N$ and $x_{i} \in X_{i}$, we define $\alpha\left(x_{i}\right)$ as the greatest in the tree order (i.e., the furthest from the origin) decision node of player $i$ that can be reached when player $i$ chooses $x_{i}$. It is well defined because of (3.6a). Now if $x_{i}$ prescribes to player $i$ to choose a 
terminal node $t$ at $\alpha\left(x_{i}\right)$, i.e., if $x_{\alpha\left(x_{i}\right)}=t \in T$, we define $p\left(x_{i}\right)=\gamma(t)$; otherwise, $p\left(x_{i}\right) \in A$ is arbitrary.

Checking (3.1) is easy. Let $\tau\left(\alpha_{0}, x_{N}\right)=t$, hence $g\left(x_{N}\right)=\gamma(t)$. We denote $i$ the player who made the last move in the play ending at $t$, and $\beta$ the decision node where that last move was made. Obviously, $\beta=\alpha\left(x_{i}\right)$ and $\gamma(t)=p\left(x_{i}\right)$.

By Proposition 3.8, every two-person perfect information game form satisfying assumptions (3.6) is simple. The converse to Proposition 3.12 is wrong: the proof only needs (3.6a). On the other hand, Proposition 3.12 becomes wrong if assumptions (3.6) are dropped altogether.

Example 3.13. Let us consider a free two-person perfect information game form with three decision nodes; we assume $D_{1}=\left\{\alpha_{0}\right\}$ and $D_{2}=\left\{\beta^{\prime}, \beta^{\prime \prime}\right\}$.

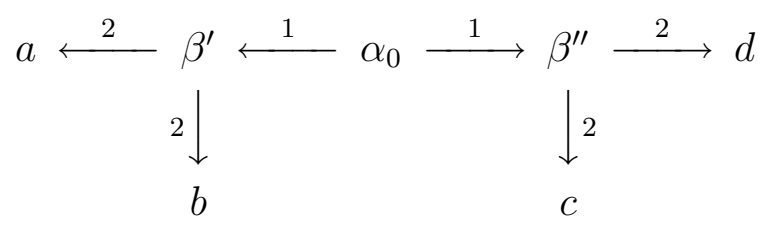

If it were a separable game form, we could, without restricting generality, assume $p\left(\beta^{\prime}\right)=a$ and $p\left(\beta^{\prime \prime}\right)=d$. Now if $p(b, c) \neq b$, then (3.1) is violated for $x_{N}=\left\langle\beta^{\prime},(b, c)\right\rangle$; if $p(b, c) \neq c$, then $(3.1)$ is violated for $x_{N}=\left\langle\beta^{\prime \prime},(b, c)\right\rangle$.

Given a perfect information game form, we define the distance between two strategies of the same player $i \in N$ as

$$
d\left(x_{i}, y_{i}\right)=\#\left\{\alpha \in D_{i} \mid y_{\alpha} \neq x_{\alpha}\right\}
$$

Then we define admissible change relations by (2.10) with the "costs" $\delta_{x_{-i}}\left(x_{i}, y_{i}\right)=d\left(x_{i}, y_{i}\right)$ for every $i \in N$ and $x_{-i} \in X_{-i}$. In other words, a change of strategy is admissible if the number of nodes involved is minimal.

Remark. If a strategy change is admissible in the sense of (3.7), then every node where a change has happened must be on the new play of the game. The converse is true for free PIGFs, but not generally. Actually, Theorem 3.14 remains valid if we define admissibility as the absence of changes at irrelevant nodes; even the same proof will do. However, it seems impossible to describe such admissibility with naturally looking "costs."

Theorem 3.14 (Theorem 3 of Kukushkin, 2002a). Every perfect information game form has an almost unrestricted FIP with admissible change relations defined by the "costs" (3.7).

Remark. A modification of the proof from Kukushkin (2002a) allows us to derive a corollary (Proposition 3.19 in the next subsection), which may be of some interest. 
Proof. Let $x_{N} \in X_{N}$ and $t \in T$; we say that $t$ is blocked by player $i$ at $x_{N}$ if there exist $\alpha \in D_{i}$ and $\beta \in X_{\alpha}$ such that $t \in \pi\left(\beta, x_{N}\right) \backslash \pi\left(\alpha, x_{N}\right)$ (it follows immediately that $\tau\left(\beta, x_{N}\right)=t$ and $\left.x_{\alpha} \neq \beta\right)$. Let $t \neq \tau\left(\alpha_{0}, x_{N}\right)$; then the set $\left\{\alpha<t \mid t \notin \pi\left(\alpha, x_{N}\right)\right\}$ is a nonempty chain, so we may pick its maximum $\alpha$ and denote $i=\nu(\alpha)$. Obviously, $t$ is blocked by $i$ at $x_{N}$ and $t$ cannot be blocked at $x_{N}$ by any other player. We thus obtain a partitioning of $T \backslash\left\{\tau\left(\alpha_{0}, x_{N}\right)\right\}$ into subsets $B_{i}\left(x_{N}\right)$ of terminal nodes blocked by each particular player $i \in N$ at $x_{N}$.

It is important to note that $\# B_{i}\left(x_{N}\right)$ does not depend on $x_{N}$; actually, $\# B_{i}\left(x_{N}\right)=$ $\left[\sum_{\alpha \in D_{i}} \# X_{\alpha}\right]-\# D_{i}$ because choosing any alternative from $X_{\alpha}$ for $\alpha \in D_{i}$, player $i$ does not choose each of $\# X_{\alpha}-1$ others thereby blocking the same number of terminal nodes (exactly which terminal nodes are blocked depends on the choices at successive nodes).

Defining $P\left(x_{N}\right)=-\sum_{i \in N} \sum_{t \in B_{i}\left(x_{N}\right)} v_{i} \circ \gamma(t)$, let us show that $P$ is a numeric potential, in the sense of $(2.5 \mathrm{a})$, of $\bowtie$. Assuming $y_{N} \gg_{i} x_{N}$, we have to prove that $P\left(y_{N}\right)>P\left(x_{N}\right)$.

We denote $t=\tau\left(\alpha_{0}, x_{N}\right)$ and $t^{\prime}=\tau\left(\alpha_{0}, y_{N}\right)$; then $v_{i} \circ \gamma\left(t^{\prime}\right)>v_{i} \circ \gamma(t)$ and $t \wedge t^{\prime} \in D_{i}$. Let us show first $B_{j}\left(x_{N}\right) \subseteq B_{j}\left(y_{N}\right)$ for each $j \neq i$. Assuming $t^{\prime \prime} \in B_{j}\left(x_{N}\right)$, we, by the definition of blocking, have $\alpha \in D_{j}$ and $\beta \in X_{\alpha}$ such that $t^{\prime \prime} \in \pi\left(\beta, x_{N}\right) \backslash \pi\left(\alpha, x_{N}\right)$. Since $j \neq i, y_{\alpha}=x_{\alpha}$, hence $t^{\prime \prime} \notin \pi\left(\alpha, y_{N}\right)$ and $t^{\prime \prime} \neq t^{\prime}$; moreover, $t^{\prime \prime} \wedge t^{\prime} \leq \alpha$. Now $t^{\prime \prime} \neq \tau\left(\beta, y_{N}\right)$ could only be possible if there were $\beta^{\prime} \in \pi\left(\beta, x_{N}\right)$ such that $y_{\beta^{\prime}} \neq x_{\beta^{\prime}}$, hence $\beta^{\prime} \in D_{i}$; but then the replacement of $x_{\beta^{\prime}}$ with $y_{\beta^{\prime}}$ would be a superfluous change incompatible with the minimization of "costs" (3.7). More formally, considering $z_{i}$ which coincides with $x_{i}$ at $\beta^{\prime}$ and with $y_{i}$ at all other nodes from $D_{i}$, we immediately see that $g\left(z_{i}, x_{-i}\right)=g\left(y_{i}, x_{-i}\right)=g\left(y_{N}\right)$ while $\delta_{x_{-i}}\left(x_{i}, z_{i}\right)<\delta_{x_{-i}}\left(x_{i}, y_{i}\right)$. Therefore, $\tau\left(\beta, y_{N}\right)=t^{\prime \prime}$, hence $t^{\prime \prime} \in B_{j}\left(y_{N}\right)$.

Now $B_{j}\left(x_{N}\right) \subseteq B_{j}\left(y_{N}\right)$ implies $B_{j}\left(x_{N}\right)=B_{j}\left(y_{N}\right)$ for all $j \neq i$. It follows immediately that $B_{i}\left(y_{N}\right)=\left(B_{i}\left(x_{N}\right) \backslash\left\{t^{\prime}\right\}\right) \cup\{t\}$, hence

$$
P\left(y_{N}\right)-P\left(x_{N}\right)=v_{i} \circ \gamma\left(t^{\prime}\right)-v_{i} \circ \gamma(t)=u_{i}\left(y_{N}\right)-u_{i}\left(x_{N}\right) .
$$

Thus, we have $P\left(y_{N}\right)>P\left(x_{N}\right)$.

An extension of the notion of a PIGF is met in the literature quite often, see, e.g., Boros and Gurvich (2003) and references therein. Suppose there is a directed graph, its nonterminal nodes are partitioned among the players, and one of them is fixed as the origin. Each player is free to choose an arc leading from each of her decision nodes ("stationary strategies"); once all strategies are chosen, a play of the game is uniquely defined: after a finite number of steps, either a terminal node is reached, or a cycle starts repeating itself ad infinitum. Treating cycles as additional outcomes, we obtain a game form.

If the players were allowed to condition their choices on history, we would return to a game on a (finite or infinite) tree. The stationarity requirement is effectively equivalent to imperfect information: when making a decision at a node, the player does not know how the play has come there. It is well known that a game with imperfect information, even on a finite tree, need not possess a Nash equilibrium (unless mixed strategies are invoked). In this class of games, however, the lack of information only concerns the past, which does 
not affect future possibilities. Therefore, it seems natural to expect no big difference with the standard perfect information model.

Indeed, if the graph is acyclic, i.e., the underlying tree is finite, an equilibrium can be obtained by the standard backward recursion. If cycles are possible, the underlying tree becomes infinite, and there is nowhere to derive an equilibrium from. An intermediate case emerges when the graph contains cycles, but every cycle is worse for each player than any terminal node (Boros and Gurvich, 2003); then no infinite play can result from any improvement.

Here we briefly consider the possibility to extend Theorem 3.14 to such a game on an acyclic graph. The result, Theorem 3.15 below, is distinctly weaker; to be more precise, a restricted FIP is established, but an almost unrestricted FIP is not (so far). Andersson et al. (2008) very recently studied the acyclicity of improvements in such game forms on graphs with or without cycles; their Theorem 2 is very close to our Theorem 3.15. However, there is a clear difference in the general approach: every notion here is applicable to any game form, while their types of improvement cycles only make sense within that class of games.

The definition of a perfect information game form on an acyclic graph is most conveniently given in the same style as in the beginning of this subsection. Instead of a game tree, we consider a finite partially ordered set $K$ satisfying condition (b) from the definition of a PIGF: the existence of the origin $\alpha_{0}$. The partition of $K$ into decision nodes $D$ and terminal nodes $T$ is the same; the ownership mapping $\nu: D \rightarrow N$ is the same; the definitions of immediate successors $X_{\alpha}$ of $\alpha \in K$, as well as strategies $X_{i}=\prod_{\alpha \in D_{i}} X_{\alpha}$ and strategy profiles $X_{N}=\prod_{i \in N} X_{i}=\prod_{\alpha \in D} X_{\alpha}$ are the same. Since condition (a) from the definition of a PIGF is dropped, we may always assume that $\gamma$ is a bijection, i.e., $T=A$. A play of the game starting at $\alpha, \pi\left(\alpha, x_{N}\right) \subseteq K$, is defined in the same way, and still is a chain; the result of playing $x_{N}$ starting at $\alpha, \tau\left(\alpha, x_{N}\right)$, is still uniquely defined. Finally, the assumption $X_{\alpha} \cap D_{i}=\emptyset$ for all $i \in N$ and $\alpha \in D_{i}$ no longer looks innocuous and is not made.

We start with the definition of a strengthened version of restricted FIP. Given a family of "costs" $\delta_{x_{-i}}$ on each $X_{i} \times X_{i}$, we define the cost-efficient individual improvement relation on $X_{N}$ by

$$
\begin{aligned}
y_{N} \gg_{i} x_{N} \rightleftharpoons\left[y_{N} \triangleright^{\text {Ind }}{ }_{i}\right. & x_{N} \& \\
& \left.\nexists z_{i} \in X_{i}\left[\delta_{x_{-i}}\left(x_{i}, z_{i}\right)<\delta_{x_{-i}}\left(x_{i}, y_{i}\right) \& u_{i}\left(z_{i}, x_{-i}\right) \geq u_{i}\left(y_{N}\right)\right]\right]
\end{aligned}
$$

and

$$
y_{N} \gg x_{N} \rightleftharpoons \exists i \in N\left[y_{N} \gg_{i} x_{N}\right] .
$$

A game form has an FCEIP if there are "costs" $\delta_{x_{-i}}$ on each $X_{i} \times X_{i}$ such that the costefficient individual improvement relation (3.9) is acyclic in every derivative game.

Theorem 3.15. Every perfect information game form on an acyclic graph has an FCEIP with the "costs" (3.7). 
Proof. Given $x_{N} \in X_{N}$ and $\alpha \in D$, we define $v_{\alpha}\left(x_{N}\right)=v_{\nu(\alpha)}\left(\tau\left(\alpha, x_{N}\right)\right)$ : what the player who moves at $\alpha$ would get if the play passes through the node. Then we define a lexicographic order on $X_{N}$ :

$$
\begin{aligned}
y_{N} \succ x_{N} \rightleftharpoons & {\left[\exists \alpha \in K\left[v_{\alpha}\left(y_{N}\right)>v_{\alpha}\left(x_{N}\right)\right] \&\right.} \\
& \left.\forall \alpha \in K\left(v_{\alpha}\left(y_{N}\right)<v_{\alpha}\left(x_{N}\right) \Rightarrow \exists \beta \in K\left[\beta>\alpha \& v_{\beta}\left(y_{N}\right)>v_{\beta}\left(x_{N}\right)\right]\right)\right] .
\end{aligned}
$$

Clearly, $\succ$ is a strict order.

Supposing that $x_{N}, y_{N} \in X_{N}$ and $y_{N} \gg_{i} x_{N}$, we show that $y_{N} \succ x_{N}$. We denote $\mathrm{B}=\left\{\beta \in K \mid y_{\beta} \neq x_{\beta}\right\} \neq \emptyset$. Exactly as in the proof of Theorem 3.14, we have $\mathrm{B} \subseteq$ $D_{i} \cap \pi\left(\alpha_{0}, y_{N}\right)$, hence there exists $\beta^{*}=\max \mathrm{B}$.

Let us show that $v_{\beta^{*}}\left(y_{N}\right)>v_{\beta^{*}}\left(x_{N}\right)$. Supposing the contrary, we define $z_{N} \in X_{N}$ by $z_{\beta^{*}}=x_{\beta^{*}}$ and $z_{\alpha}=y_{\alpha}$ for all $\alpha \neq \beta^{*}$. Clearly, $u_{i}\left(z_{N}\right)=v_{\beta^{*}}\left(z_{N}\right)=v_{\beta^{*}}\left(x_{N}\right) \geq v_{\beta^{*}}\left(y_{N}\right)=$ $u_{i}\left(y_{N}\right)$. Since $d\left(x_{i}, z_{i}\right)<d\left(x_{i}, y_{i}\right)$, we have a contradiction with the assumption $y_{N} \gg_{i} x_{N}$.

Finally, let $\alpha \in K$ and $v_{\alpha}\left(y_{N}\right) \neq v_{\alpha}\left(x_{N}\right)$, hence $\mathrm{B} \cap \pi\left(\alpha, y_{N}\right) \neq \emptyset$. Picking $\beta \in$ $\mathrm{B} \cap \pi\left(\alpha, y_{N}\right)$, we have $\beta^{*} \geq \beta \geq \alpha$. Since $v_{\beta^{*}}\left(y_{N}\right)>v_{\beta^{*}}\left(x_{N}\right)$, (3.10) holds.

It is impossible to derive an almost unrestricted FIP from the above argument: the inequality $v_{\beta^{*}}\left(y_{N}\right)>v_{\beta^{*}}\left(x_{N}\right)$ need not hold without the "cost-efficiency" of the improvement. Whether the conditions of Theorem 3.15 imply the property itself remains an open question.

Proposition 3.16. A perfect information game form on an acyclic graph has an almost unrestricted FIP with admissible change relations defined by the "costs" (3.7) if each $D_{i}$ is an anti-chain, i.e., all $\beta, \beta^{\prime} \in D_{i}$ are incomparable in the order on $K$.

Proof. Let $y_{N} \gg_{i} x_{N}$. In the notation from the proof of Theorem 3.15, we have $\mathrm{B}=\left\{\beta^{*}\right\}$, hence $v_{\beta^{*}}\left(y_{N}\right)=u_{i}\left(y_{N}\right)>u_{i}\left(x_{N}\right)=v_{\beta^{*}}\left(x_{N}\right)$, hence $y_{N} \succ x_{N}$.

It is funny to contrast Proposition 3.16 with Theorem 3.9.

Proposition 3.17. A perfect information game form on an acyclic graph has the FIP if each $D_{i}$ is a singleton.

Proof. Retaining the notation from the proof of Theorem 3.15 and arguing in the same way, it is easy to show that $y_{N} \succ x_{N}$ whenever $y_{N} \triangleright^{\text {Ind }} x_{N}$.

The converse to Proposition 3.17 is wrong. There is no plausible conjecture about necessary and sufficient conditions for FIP in this class of game forms.

\subsection{Cardinal utilities and potentials}

Although our main subject are games with ordinal preferences and we do not consider mixed extensions, it seems impossible not to say a few words about cardinal utilities. Actually, Monderer and Shapley (1996) paid most attention to that case. Moreover, they assumed 
that the utilities of all players are measured in the same scale; we make the same assumption here.

Let $\Gamma$ be a game with such "co-cardinal" utilities. Monderer and Shapley (1996) defined an exact potential of $\Gamma$ as a function $P: X_{N} \rightarrow \mathbb{R}$ such that

$$
u_{i}\left(y_{N}\right)-u_{i}\left(x_{N}\right)=P\left(y_{N}\right)-P\left(x_{N}\right)
$$

whenever $i \in N, y_{N}, x_{N} \in X_{N}$, and $y_{-i}=x_{-i}$. An ordinal potential of $\Gamma$ is a function $P: X_{N} \rightarrow \mathbb{R}$ such that

$$
\operatorname{sign}\left(u_{i}\left(y_{N}\right)-u_{i}\left(x_{N}\right)\right)=\operatorname{sign}\left(P\left(y_{N}\right)-P\left(x_{N}\right)\right)
$$

whenever $i \in N, y_{N}, x_{N} \in X_{N}$, and $y_{-i}=x_{-i}$. The latter notion is indeed ordinal, i.e., invariant under strictly increasing transformations of utilities. Voorneveld and Norde (1996) showed that the existence of an ordinal potential is equivalent to the absence of "weak improvement cycles."

Obviously, (3.11) implies (3.12), of which it is the most natural cardinal analogue.

Theorem 3.18. For every game form $G$, the following statements are equivalent.

1. Every derivative game $G\left(v_{N}\right)$ admits an exact potential.

2. Every derivative game $G\left(v_{N}\right)$ admits an ordinal potential.

3. $G$ is dictatorial.

Proof. The implications [Statement $3 \Rightarrow$ Statement $1 \Rightarrow$ Statement 2] are straightforward. Let Statement 2 hold; then $G$ has the FIP. Let $x_{N} \in X_{N}, i \in N$, and $y_{i} \in X_{i}$; we say that $y_{i}$ is effective at $x_{N}$ if $g\left(y_{i}, x_{-i}\right) \neq g\left(x_{N}\right)$.

Step 3.18.1. Let $x_{N} \in X_{N}$. If a player has a strategy effective at $x_{N}$, then no strategy of any other player is effective at $x_{N}$.

Proof. Suppose the contrary: two players have effective strategies at the same strategy profile. Taking into account Proposition 3.4, we see that $G$ must contain a $2 \times 2$ fragment of the type $\begin{array}{cc}a & c \\ b & b\end{array}$ with $b \neq a \neq c$. Assuming, without restricting generality, that $i$ chooses rows and $j$ columns, we consider a utility vector $v_{N}$ such that $v_{i}(a)>v_{i}(b) \geq v_{i}(c)$ and $v_{j}(c)>v_{j}(a)$. Clearly, $G\left(v_{N}\right)$ cannot admit an ordinal potential.

Step 3.18.2. If $y_{i}$ is effective at $x_{N}, z_{i} \in X_{i}, j \in N \backslash\{i\}$ and $z_{j} \in X_{j}$, then $z_{j}$ is not effective at $\left(z_{i}, x_{-i}\right)$.

Proof. We have either $g\left(z_{i}, x_{-i}\right) \neq g\left(x_{N}\right)$ or $g\left(z_{i}, x_{-i}\right) \neq g\left(y_{i}, x_{-i}\right)$, hence either $x_{i}$ or $y_{i}$ is effective at $\left(z_{i}, x_{-i}\right)$. Now Step 3.18.1 applies.

Step 3.18.3. If $y_{i}$ is effective at $x_{N}$, then player $i$ is a dictator. 
Proof. For every $z_{i} \in X_{i}$, we define $p\left(z_{i}\right)=g\left(z_{i}, x_{-i}\right)$. By Step 3.18.2, $g\left(z_{i}, z_{-i}\right)=p\left(z_{i}\right)$ whenever $z_{-i}$ differs from $x_{-i}$ in the choice of one player, i.e., given $j \in N \backslash\{i\}$ and $z_{j} \in X_{j}$, we have $g\left(x_{i}, z_{j}, x_{-i j}\right)=p\left(x_{i}\right) \neq p\left(y_{i}\right)=g\left(y_{i}, z_{j}, x_{-i j}\right)$. Therefore, $y_{i}$ is effective at $\left(x_{i}, z_{j}, x_{-i j}\right)$, so the same Step 3.18.2 applies again and we have $g\left(z_{i}, z_{-i}\right)=p\left(z_{i}\right)$ whenever $z_{-i}$ differs from $x_{-i}$ in the choice of two players. Iterating the reasoning, we see that $g\left(z_{i}, z_{-i}\right)=p\left(z_{i}\right)$ for all $z_{-i} \in X_{-i}$, i.e., player $i$ is a dictator.

If $\# g\left(X_{N}\right)=1$, then every player is a dictator. If $g\left(x_{N}\right) \neq g\left(y_{N}\right)$, there is a sequence $x_{N}^{0}, x_{N}^{1}, \ldots, x_{N}^{n}$ such that $x_{N}^{0}=x_{N}, x_{N}^{n}=y_{N}$, and $x_{-i(k)}^{k+1}=x_{-i(k)}^{k}$ for each $k \in\{0, \ldots, n-1\}$ and some $i(k) \in N$. Clearly, we must have $g\left(x_{N}^{k}\right) \neq g\left(x_{N}^{k+1}\right)$ for some $k$, hence Step 3.18 .3 applies.

Remark. Theorem 3.18 remains valid if Statements 1 and 2 are restricted to injective utilities (i.e., strict preferences): If $b \neq c$ in Step 3.18.1, then we may assume $v_{i}(b)>v_{i}(c)$.

We call $G$ a nearly potential game form if there is a liberal admissible change relation $\vdash_{i}$ on $X_{N}$ for each $i \in N$, and a function $P: X_{N} \rightarrow \mathbb{R}$ for every derivative game $G\left(v_{N}\right)$ such that (3.11) holds whenever $i \in N, y_{N}, x_{N} \in X_{N}, x_{N} \vdash_{i} y_{N}$, and $g\left(y_{N}\right) \neq g\left(x_{N}\right)$. We call $G$ an almost potential game form if there is a function $P: X_{N} \rightarrow \mathbb{R}$ for every derivative game $G\left(v_{N}\right)$ such that (3.11) holds whenever $i \in N, y_{N}, x_{N} \in X_{N}, x_{-i}=y_{-i}$, and $g\left(y_{N}\right) \neq g\left(x_{N}\right)$. Clearly, every almost potential game form has the FIP while every nearly potential game form has an almost unrestricted FIP.

Proposition 3.19. Every perfect information game form is a nearly potential one. If $\nu$ is injective, i.e., each player has at most one decision node, then it is an almost potential game form.

Proof. Defining the admissible change relations by the "costs" (3.7) and potential $P$ as in the proof of Theorem 3.14, we refer to (3.8). If each $D_{i}$ is a singleton, then every change of strategy producing a change of outcome is admissible.

Proposition 3.20. If a free perfect information game form is an almost potential one, then $\nu$ is injective.

Proof. By Theorem 3.9, each $D_{i}$ is a chain, so if $\nu$ is not injective, we must have $\nu(\alpha)=$ $\nu\left(\alpha^{\prime}\right)=i$ while $\alpha<\alpha^{\prime}$. By the non-degeneracy assumptions, there must be $\beta \in D_{j}$ such that $\alpha<\beta<\alpha^{\prime}$ and $j \neq i$. Since $G$ is free, there must be a fragment of the type

$$
\begin{array}{lll}
a & b & b \\
a & c & d
\end{array}
$$

where player $i$ chooses columns and player $j$ rows, and different letters denote different outcomes. Individual changes of strategies starting at the northwestern corner produce this cycle of outcomes: $a \rightarrow b \rightarrow c \rightarrow d \rightarrow b \rightarrow a$. Therefore, (3.11) would imply that $v_{i}(b)-v_{i}(a)+v_{j}(c)-v_{j}(b)+v_{i}(d)-v_{i}(c)+v_{j}(b)-v_{j}(d)+v_{i}(a)-v_{i}(b)=0$, hence $v_{i}(d)-v_{i}(c)=v_{j}(d)-v_{j}(c)$. Clearly, the equality does not hold for all utility functions. 
The assumption that the game form is free cannot be dropped. Example 4.11 below disproves putative hypotheses like "every nearly (or almost) potential game form can be represented as a perfect information game form"; on the other hand, such examples seem rare, so a plausible conjecture may be obtainable.

\section{FCP Game Forms}

\subsection{Characterization}

Given $I \in \mathcal{N} \backslash\{N\}$, a superfragment of $G$ is a game form with two players, "I" and " $-I$," nonempty subsets of $X_{I}$ and $X_{-I}$ as strategy sets, respectively, and the appropriate restriction of $g$. The $\mathrm{FCP}\left({ }^{+}\right)$of $G$ obviously implies the same property of every superfragment; no such assertion holds for the FIP.

A game form $G$ is supertight if every $2 \times 2$ superfragment of $G$ contains a simple strategy; in other words, if the condition

$$
\left\{g\left(x_{I}^{\prime}, x_{-I}^{\prime}\right), g\left(x_{I}^{\prime \prime}, x_{-I}^{\prime \prime}\right)\right\} \cap\left\{g\left(x_{I}^{\prime}, x_{-I}^{\prime \prime}\right), g\left(x_{I}^{\prime \prime}, x_{-I}^{\prime}\right)\right\} \neq \emptyset
$$

holds for every $I \in \mathcal{N}, x_{I}^{\prime}, x_{I}^{\prime \prime} \in X_{I}$ and $x_{-I}^{\prime}, x_{-I}^{\prime \prime} \in X_{-I}$.

Remark. Boros et al. (2010) call such game forms "totally tight"; again, a lone adjective seems preferable. Boros et al. (2009) proved that every supertight game form is separable.

Proposition 4.1. Let $G$ be a game form such that $g\left(X_{N}\right)=\{q, a\}$. Then $G$ is supertight if and only if there is an ordering $\succeq_{I}$ on $X_{I}$ for every $I \in \mathcal{N}$ such that

$$
\forall I \in \mathcal{N} \forall x_{N}, x_{N}^{\prime} \in X_{N}\left[\left[g\left(x_{N}^{\prime}\right)=q \& x_{I}^{\prime} \succeq_{I} x_{I} \& x_{-I}^{\prime}=x_{-I}\right] \Rightarrow g\left(x_{N}\right)=q\right] .
$$

An interpretation should be clear: all strategies of a player or a coalition are (weakly) ordered according to how much effort is spent in trying to switch from a status quo ante outcome $q$ to the unique available alternative; whenever the effort proves insufficient, less effort cannot be sufficient. The term "a binary lobbing game form" may be appropriate.

Proof. Necessity. Let (4.1) hold everywhere. For each $I \in \mathcal{N}$, we define a binary relation on $X_{I}$ :

$$
y_{I} \succeq_{I} x_{I} \rightleftharpoons \forall x_{-I} \in X_{-I}\left[g\left(y_{I}, x_{-I}\right)=q \Rightarrow g\left(x_{I}, x_{-I}\right)=q\right] ;
$$

the relation is obviously a preorder. Incomparability of $y_{I}$ and $x_{I}$ would immediately imply a violation of (4.1); therefore, $\succeq_{I}$ is an ordering for every $I \in \mathcal{N}$. The "monotonicity" requirement (4.2) immediately follows from (4.3).

Sufficiency. Suppose that (4.2) holds, but (4.1) is violated by a superfragment. Without restricting generality, $x_{I}^{\prime \prime} \succeq_{I} x_{I}^{\prime}$ and $x_{-I}^{\prime \prime} \succeq_{-I} x_{-I}^{\prime}$; therefore, $g\left(x_{I}^{\prime}, x_{-I}^{\prime}\right)=q$. The supposed negation of (4.1) implies that $g\left(x_{I}^{\prime \prime}, x_{-I}^{\prime}\right)=g\left(x_{I}^{\prime}, x_{-I}^{\prime \prime}\right)=a$, but then $g\left(x_{I}^{\prime \prime}, x_{-I}^{\prime \prime}\right)=a$ by $(4.2)$. 
Theorem 4.2. A game form $G$ has the FCP if and only if $G$ is either dictatorial, or supertight with $\# g\left(X_{N}\right) \leq 2$.

Remark. So far, there is no explanation for the obvious similarity with Arrow's impossibility theorem.

Proof. Sufficiency. A dictatorial game form poses no problem. Let $G$ be supertight with $g\left(X_{N}\right)=\{a, q\}$ and $x_{N}^{0}, x_{N}^{1}, \ldots, x_{N}^{m}=x_{N}^{0}$ be a coalition improvement cycle in a derivative game $G\left(v_{N}\right)$. Without restricting generality, $g\left(x_{N}^{0}\right)=a$, hence $g\left(x_{N}^{2 k}\right)=a, g\left(x_{N}^{2 k+1}\right)=q$, and $m$ is even. We denote $I=\left\{i \in N \mid v_{i}(a)>v_{i}(q)\right\}, J=\left\{i \in N \mid v_{i}(q)>v_{i}(a)\right\}$, and $N^{0}=\left\{i \in N \mid v_{i}(a)=v_{i}(q)\right\}$. Each player $i \in N^{0}$ chooses the same strategy at each $x_{N}^{k}$. Obviously, $x_{I}^{2 k+1}=x_{I}^{2 k}$ and $x_{-I}^{2 k+2}=x_{-I}^{2 k+1}$ for all $k$. By Proposition 4.1, we have $x_{I}^{2 k+2} \succ_{I} x_{I}^{2 k+1}=x_{I}^{2 k}$ for each $k$, which contradicts the assumption $x_{N}^{m}=x_{N}^{0}$.

Necessity. Let $G$ have the FCP; then (4.1) holds by Proposition 3.4.

Step 4.2.1. $\# g\left(X_{N}^{\prime}\right) \leq 2$ for every $2 \times 2$ superfragment of $G$.

Proof. Suppose the contrary: there is a superfragment of $G$ of the type ${ }_{d}^{a} b$ with $a \neq b \neq$ $c \neq a$. Let $I$ choose rows and $-I$ columns. We consider a utility vector $v_{N}$ such that $v_{i}(a)>v_{i}(c)>v_{i}(b)$ for each $i \in I$ and $v_{i}(b)>v_{i}(a)>v_{i}(c)$ for each $i \notin I$. A coalition improvement cycle in $G\left(v_{N}\right)$ is obvious: $a \rightarrow b \rightarrow c \rightarrow a$.

Suppose that $G$ is not dictatorial. Then for each $i \in N$ there is $x_{i} \in X_{i}$ such that $\# g\left(x_{i}, X_{-i}\right) \geq 2$. Moreover, at least two players are not "dummies," i.e., there is $x_{-i} \in X_{-i}$ such that $\# g\left(X_{i}, x_{-i}\right) \geq 2$. Whenever $i \in N, x_{i}, x_{i}^{\prime} \in X_{i}, x_{-i}, x_{-i}^{\prime} \in X_{-i}$ and $g\left(x_{i}^{\prime}, x_{-i}\right) \neq g\left(x_{i}, x_{-i}\right) \neq g\left(x_{i}, x_{-i}^{\prime}\right)$, there holds $g\left(x_{i}^{\prime}, x_{-i}\right)=g\left(x_{i}, x_{-i}^{\prime}\right)$ by Step 4.2.1. It follows immediately that $\# g\left(x_{i}, X_{-i}\right) \leq 2$ for every non-dummy $i \in N$ and $x_{i} \in X_{i}$.

If $\# g\left(X_{N}\right)>2$, there must exist $i \in N, x_{i}, x_{i}^{\prime} \in X_{i}$ and $a, b, c, d \in A$ such that $a \neq b \neq c \neq a, b \neq d \neq c, g\left(x_{i}, X_{-i}\right)=\{a, b\}$, and $g\left(x_{i}^{\prime}, X_{-i}\right)=\{c, d\}$. We pick $x_{-i} \in X_{-i}$ such that $g\left(x_{i}, x_{-i}\right)=b$. If $g\left(x_{i}^{\prime}, x_{-i}\right)=d$, we pick $x_{-i}^{\prime} \in X_{-i}$ such that $g\left(x_{i}^{\prime}, x_{-i}^{\prime}\right)=c$ and obtain a contradiction with Step 4.2.1. If $g\left(x_{i}^{\prime}, x_{-i}\right)=c$, we pick $x_{-i}^{\prime} \in X_{-i}$ such that $g\left(x_{i}^{\prime}, x_{-i}^{\prime}\right)=d$ and again obtain a contradiction with Step 4.2.1.

Theorem 4.3. A game form $G$ has the $F C P^{+}$if and only if it has the FCP and either $\# g\left(X_{N}\right)=1$ or $\# N \leq 2$.

Proof. Sufficiency. Let $\# N=2$. Given a utility vector $v_{N}$, we take $v_{N}^{*}$ with the property

$$
v_{i}^{*}(a)>v_{i}^{*}(b) \Longleftrightarrow\left[v_{i}(a)>v_{i}(b) \text { or }\left[v_{i}(a)=v_{i}(b) \& v_{-i}(a)>v_{-i}(b)\right]\right]
$$

for each $i \in N$ and $a, b \in A$. Clearly, a weak coalition improvement path in $G\left(v_{N}\right)$ is a coalition improvement path in $G\left(v_{N}^{*}\right)$, hence $G$ has the $\mathrm{FCP}^{+}$as well as the FCP.

Necessity. Let $\# g\left(X_{N}\right)>1$; then there are $i \in N x_{i}, x_{i}^{\prime} \in X_{i}$, and $x_{-i} \in X_{-i}$ such that $a=g\left(x_{i}, x_{-i}\right) \neq g\left(x_{i}^{\prime}, x_{-i}\right)=b$. Supposing $\# N>2$, we can pick $j, k \in N$ such that $j \neq i \neq k \neq j$. Whenever $v_{i}(a)=v_{i}(b), v_{j}(a)>v_{j}(b)$, and $v_{k}(a)<v_{k}(b)$, we have a weak coalition improvement cycle where player $i$ switches between $x_{i}$ and $x_{i}^{\prime}$ back and forth, while the other players choose $x_{-i}$. 


\subsection{Voting by veto}

The title of this subsection refers to a class of voting procedures; each of them defines a game form. There are finite sets of players $N$ and of outcomes, or alternatives, $A$. The players may have arbitrary preferences over the outcomes. A voting by veto procedure specifies positive integer numbers $\lambda_{a}$ and $\mu_{i}$ for each $a \in A$ and $i \in N: \mu_{i}$ is the number of black balls given to player $i$; $\lambda_{a}$ is the "veto-resistance" of outcome $a$. Each player allocates his/her black balls among the outcomes; if the number of balls allocated to $a \in A$ equals or exceeds $\lambda_{a}$, the outcome is vetoed. To ensure the existence of non-vetoed outcomes, we impose the restriction

$$
\sum_{a \in A} \lambda_{a} \geq \sum_{i \in N} \mu_{i}+1
$$

A linear order is assumed on $A$; if several outcomes are not vetoed, the highest of them is selected.

To describe strategies formally, we assume there is a finite set $D$ of black balls with an ownership mapping $\nu: D \rightarrow N$. Balls from $D_{i}=\nu^{-1}(i)$ are given to player $i$; we assume $\# D_{i}=\mu_{i}$. A strategy of player $i$ is a mapping $x_{i}: D_{i} \rightarrow A$; a strategy profile can be understood as a mapping $x_{N}: D \rightarrow A$. For each $I \in \mathcal{N}$, we denote $D_{I}=\bigcup_{i \in I} D_{i}$; every strategy of the coalition can be understood as a mapping $x_{I}: D_{I} \rightarrow A$. Given $a \in A$, $I \in \mathcal{N}$, and $x_{I} \in X_{I}$, we denote $\varkappa\left(a, x_{I}\right)=\# x_{I}^{-1}(a)$, the number of balls cast to $a$ by $I$ under $x_{I}$. An outcome $a$ is vetoed at a strategy profile $x_{N}$ if $\varkappa\left(a, x_{N}\right) \geq \lambda_{a}$, and over-vetoed if the inequality is strict. $g\left(x_{N}\right)$ is the highest non-vetoed outcome.

Remark. If there is $b \in A$ such that $\sum_{a>b} \lambda_{a} \geq \sum_{i \in N} \mu_{i}+1$, then $b \notin g\left(X_{N}\right)$. In a sense, the elimination of such outcomes would not change the game form; however, their presence creates no difficulties either.

For brevity, a game form generated by a voting by veto procedure is called a $V V$ game form.

Two potential extensions of the notion may be worth mentioning. First, we might introduce a distinction between "outcomes" from $A$ and "voting proposals," the same outcome being implied by several different proposals (similarly to the notion of a non-free perfect information game form). Our "positive" results, naturally, remain valid in this situation, but "negative" ones need not. Since the latter are just examples, the matter seems not to deserve much attention. Secondly, we might allow non-integer $\mu$ 's and $\lambda$ 's: instead of black balls, each player is given a certain amount of tar to be allocated among the outcomes. However, such voting procedures may seem too exotic, and are outside the realm of finite game forms anyway.

Proposition 4.4. Every ordered voting game form $G$ with integer $\mu$ 's and $\lambda$ 's can be represented as the fragment of a VV game form $\bar{G}$.

Proof. We retain the same set of outcomes $A$ with the same order. Denoting $m=\# A-1$, $a^{-}=\min A$, and $a^{+}=\max A$, we define veto-resistance in $\bar{G}$ as $\bar{\lambda}_{a}=\lambda_{a}$ for $a \neq a^{-}$and $\bar{\lambda}_{a^{-}}=m \cdot \sum_{i \in N} \mu_{i}+1$; each player $i$ is given $\bar{\mu}_{i}=m \cdot \mu_{i}$ black balls. Inequality (4.4) is 
obvious. Every strategy $x_{i} \in A$ in $G$ corresponds to this strategy in $\bar{G}$ : put $\mu_{i}$ balls at each $a \in\left[x_{i}+1, \ldots, a^{+}\right]$and all the rest at $a^{-}$(if $x_{i}=a^{+}$, put all the balls at $a^{-}$). It is easily checked that the highest non-vetoed outcome when all players use such strategies is the same as $g\left(x_{N}\right)$ defined by (3.4).

Proposition 4.5. Every free perfect information game form $G$ can be represented as the fragment of a $V V$ game form $\bar{G}$.

Proof. For each $\alpha \in D$, we define $\alpha^{\uparrow}=\{a \in T \mid a>\alpha\}$ and $m_{\alpha}=\# \alpha^{\uparrow}$. Then we define $\bar{A}=T \cup\left\{\alpha_{0}\right\}$ and order $\bar{A}$ in an arbitrary way with the only restriction that the relations $\alpha_{0}<a$ for every $a \in T$ are retained. Finally, we define $\bar{\mu}_{i}=\sum_{\alpha \in D_{i}} m_{\alpha}$ for each $i \in N, \bar{\lambda}_{a}=1$ for all $a \in T$, and $\bar{\lambda}_{\alpha_{0}}=\sum_{i \in N} \bar{\mu}_{i}+1$. Inequality (4.4) is again obvious. The choice of $\beta \in X_{\alpha}\left(\alpha \in D_{i}\right)$ in $G$ corresponds, in $\bar{G}$, to putting a black ball at each $a \in \alpha^{\uparrow} \backslash \beta^{\uparrow}$, and putting all the remaining (of $m_{\alpha}$ ) balls at $\alpha_{0}$. It is easily checked that when all players use such strategies, just two outcomes from $\bar{A}$ remain non-vetoed: $\alpha_{0}$ and $\tau\left(\alpha_{0}, x_{N}\right)=g\left(x_{N}\right)$.

An analog of Proposition 4.5 for non-free perfect information game forms holds if we allow "non-free" VV game forms; the proof is essentially the same. If we just drop the assumption that $G$ is free, the proposition will not survive.

Example 4.6. Let us consider a two-person perfect information game form with three decision nodes; we assume $D_{1}=\left\{\alpha_{0}\right\}$ and $D_{2}=\left\{\beta^{\prime}, \beta^{\prime \prime}\right\}$.

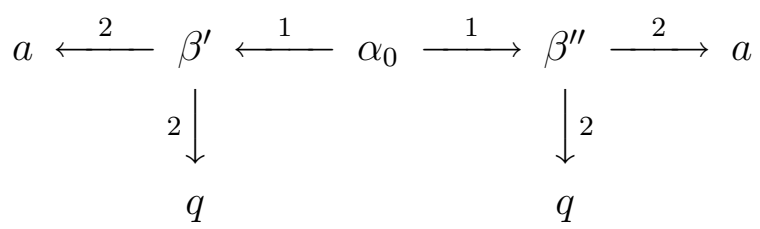

Supposing that it can be represented as the fragment of a VV game form, we may assume $q>a$ by symmetry.

The number of black balls allocated by player 1 to $q$ under the choice of $\beta^{\prime}\left(\beta^{\prime \prime}\right)$ is denoted $m_{1}^{\prime}\left(m_{1}^{\prime \prime}\right)$; without restricting generality, $m_{1}^{\prime} \geq m_{1}^{\prime \prime}$. Let us denote $\bar{x}_{2}$ the strategy of player 2 that prescribes the choice of $q$ at $\beta^{\prime}$ and $a$ at $\beta^{\prime \prime}$. The number of black balls allocated to $q$ under $\bar{x}_{2}$ is denoted $m_{2}$. Since $g\left(\beta^{\prime \prime}, \bar{x}_{2}\right)=a$, we have $m_{1}^{\prime \prime}+m_{2} \geq \lambda_{q}$; but then $m_{1}^{\prime}+m_{2} \geq \lambda_{q}$ as well, contradicting $g\left(\beta^{\prime}, \bar{x}_{2}\right)=q$.

Proposition 4.7. Every simple game form $G$ can be represented as the fragment of a $V V$ game form $\bar{G}$.

Proof. Taking into account Proposition 3.8, we may assume that $G$ is a perfect information game form where $D$ is a chain. We define $\bar{A}=A \cup\left\{\alpha_{0}\right\}$ and order $\bar{A}$ in an arbitrary way with the only restriction that $\alpha_{0}<a$ for every $a \in A$. For each $\alpha \in D$ and $a \in A$, we define $m(\alpha, a)=\#\left[\gamma^{-1}(a) \cap X_{\alpha}\right]$, i.e., the number of ways to end the play at $\alpha$ producing the 
outcome $a$. Then, again for each $\alpha \in D$ and $a \in A$, we define an integer number $\eta(\alpha, a)$ by backward recursion:

$$
\eta(\alpha, a)=m(\alpha, a)+\sum_{\beta>\alpha} \eta(\beta, a)
$$

For each $a \in A$, we define $\bar{\lambda}_{a}=\eta\left(\alpha_{0}, a\right) ; \bar{\lambda}_{\alpha_{0}}=1+\sum_{a \in A} \sum_{\alpha>\alpha_{0}} \eta(\alpha, a)$. For each $i \in$ $N$, we define $\bar{\mu}_{i}=\sum_{\alpha \in D_{i}} \sum_{a \in A} \eta(\alpha, a)$. Now we have $\sum_{i \in N} \bar{\mu}_{i}=\sum_{a \in A} \sum_{\alpha \in D} \eta(\alpha, a)=$ $\sum_{a \in A}\left[\eta\left(\alpha_{0}, a\right)+\sum_{\alpha>\alpha_{0}} \eta(\alpha, a)\right]=\sum_{a \in \bar{A}} \bar{\lambda}_{a}-1$, i.e., (4.4) holds as an equality.

Let us describe how the strategies in $G$ are to be interpreted in $\bar{G}$. The definition of $\bar{\mu}_{i}$ suggests perceiving each $\bar{D}_{i}$ as $\bigcup_{\alpha \in D_{i}} \bar{D}_{\alpha}$ with $\# \bar{D}_{\alpha}=\sum_{a \in A} \eta(\alpha, a)$; every $\bar{D}_{\alpha}$ is partitioned into $\bigcup_{a \in A} \bar{D}_{\alpha}(a)$ with $\# \bar{D}_{\alpha}(a)=\eta(\alpha, a)$, each ball from $\bar{D}_{\alpha}(a)$ having a "preordained destination" $a$. Furthermore, there is a subset $M(\alpha, a) \subseteq \bar{D}_{\alpha}(a)$ with $\# M(\alpha, a)=m(\alpha, a)$; we assume a bijection between $M(\alpha, a)$ and $\gamma^{-1}(a) \cap X_{\alpha}$ fixed.

The player moving at $\alpha \in D$ has two broad alternatives: either end the play here (there are $m(\alpha, a)$ choices leading to each outcome $a \in A$ ), or continue (a unique choice, absent if $\alpha=\max D)$. We interpret each choice at $\alpha$ as an allocation of balls from $\bar{D}_{\alpha}$ among $a \in \bar{A}$. If the decision is to continue, then $m(\alpha, a)+\sum_{\beta>\alpha+1} \eta(\beta, a)$ balls from $\bar{D}_{\alpha}(a)$ go to each $a$; all the rest goes to $\alpha_{0}$. If the decision is to finish at $\beta \in X_{\alpha}$ with $\gamma(\beta)=a$, then each ball from $\bar{D}_{\alpha}(b)(b \neq a)$ goes to $b$; each ball from $M(\alpha, a)$ except that corresponding to $\beta$ goes to $a$; all the rest goes to $\alpha_{0}$ again. Obviously, different choices in $G$ generate different allocations in $\bar{G}$.

Finally, let us consider an arbitrary decision profile in $G$. Let the decision at each $\beta<\alpha \in D$ be to continue, whereas the decision at $\alpha$ be to finish at $\alpha^{\prime} \in X_{\alpha}$ with $\gamma\left(\alpha^{\prime}\right)=a^{*}$. In $G$, we have $g\left(x_{N}\right)=a^{*}$ regardless of the choices at $\beta>\alpha$. Looking at what outcomes are vetoed in $\bar{G}$ under the corresponding strategy profile, we may assume that the players move the balls from $\bar{D}_{\beta}$ 's consecutively, in accordance with the order on $D$. Before the choice at $\alpha_{0}$ is made, there are $\bar{\lambda}_{a}=\eta\left(\alpha_{0}, a\right)=m\left(\alpha_{0}, a\right)+\sum_{\beta>\alpha_{0}} \eta(\beta, a)$ "empty slots" at each $a \in A$; the decision to continue the play fills $m\left(\alpha_{0}, a\right)+\sum_{\beta>\alpha_{0}+1} \eta(\beta, a)$ of them. Therefore, after the choice at $\alpha_{0}$ has been made, there are $\eta\left(\alpha_{0}+1, a\right)$ empty slots at each $a \in A$. A straightforward inductive argument shows that there are $\eta(\alpha, a)$ empty slots at each $a \in A$ when the choice at $\alpha$ is being made. After the decision to end the play, $\eta(\alpha, a)$ balls go to each $a \neq a^{*}$, hence they are all vetoed regardless of decisions at successive nodes. As to $a^{*}$, there remain $1+\sum_{\beta>\alpha} \eta\left(\beta, a^{*}\right)$ empty slots there; every $\beta>\alpha$ adds at most $\eta\left(\beta, a^{*}\right)$ balls. Therefore, $a^{*}$ is the highest non-vetoed outcome.

Proposition 4.8. Let $\bar{G}$ be a $V V$ game form; let $G$ be a fragment of $\bar{G}$ such that $\# g\left(X_{N}\right) \leq$ 2. Then $G$ has the FCP.

Proof. Without restricting generality, we assume $g\left(X_{N}\right)=\{q, a\}$ with $q>a$, hence $g\left(x_{N}\right)=$ $a$ if and only if $q$ is vetoed at $x_{N}$. For each $I \in \mathcal{N}$ and $x_{I}, x_{I}^{\prime} \in X_{I}$, we define $x_{I}^{\prime} \succeq_{I} x_{I} \rightleftharpoons$ $\varkappa\left(q, x_{I}^{\prime}\right) \geq \varkappa\left(q, x_{I}\right)$. It is clear now that $G$ satisfies $(4.2)$, hence the sufficiency part of Theorem 4.2 applies. 
Example 4.9. Let $A=\{a, b, c\}, a>b>c, N=\{1,2\}$, and every $\mu$ and $\lambda$ be equal to 1 . The game form is described by the following matrix:

$$
\begin{array}{ccc}
\underline{b} & \underline{c} & b \\
\underline{c} & \underline{a} & a \\
b & a & a
\end{array}
$$

Assuming $v_{1}(a)=v_{1}(b)=1, v_{1}(c)=0, v_{2}(a)=v_{2}(b)=0, v_{2}(c)=1$, we see that the underlined outcomes form an improvement cycle in $G\left(v_{N}\right)$; therefore, $G$ does not have even the FIP (although it is strongly consistent).

The example shows that fragments of VV game forms do not generally possess any nice property: the underlined $2 \times 2$ fragment is not even Nash consistent. It carries a subtler message as well. If we allowed non-free VV game forms, we might assume that $a$ and $b$ are just different proposals implying the same outcome $\left(v_{i}(a)=v_{i}(b)\right.$ for both $i$ in the example), hence $\# A=2$. Therefore, for Proposition 4.8 to survive, the restriction should be put on the number of proposals rather than outcomes.

Theorem 4.10. Let $G$ be a game form with $N=\{1,2\}$ and $A=\{q, a\}$. Then the following statements are equivalent.

1. G has the FCP.

2. $G$ is simple.

3. $G$ can be represented as the fragment of a $V V$ game form.

Proof. Suppose Statement 1 holds, hence Theorem 4.2 applies. If $G$ is dictatorial, then both Statements 2 and 3 are obvious. Otherwise, we define an ordering $\succeq_{i}$ on each $X_{i}$ by (4.3). We pick $x_{i}^{-}$and $x_{i}^{+}$(for each $i \in N$ ) among, respectively, the least and the greatest strategies in $X_{i}$ w.r.t. $\succeq_{i}$. Let us show that $\mathcal{X}$ contains a simple strategy. Pick $i \in N$; if $g\left(x_{i}^{-}, x_{-i}^{+}\right)=q$, then $g\left(x_{i}^{-}, x_{-i}\right)=q$ for all $x_{-i} \in X_{-i}$, and we are home; if $g\left(x_{i}^{-}, x_{-i}^{+}\right)=a$, then $g\left(x_{i}, x_{-i}^{+}\right)=a$ for all $x_{i} \in X_{i}$, and we are home again. Since every fragment of an FCP game form has the FCP itself, Proposition 3.2 applies, i.e., Statement 2 holds.

The implications Statement $2 \Rightarrow$ Statement 3 and Statement $3 \Rightarrow$ Statement 1 immediately follow from Propositions 4.7 and 4.8, respectively. Note that the assumption $n=2$ is not needed in either case.

Example 4.11. Let $N=\{1,2,3\}$, player 1 choose rows, player 2 columns, and player 3 matrices.

$$
\left[\begin{array}{ll}
q & a \\
q & q
\end{array}\right] \quad\left[\begin{array}{ll}
a & a \\
q & a
\end{array}\right]
$$

Condition (4.1) obviously holds, but there is no simple strategy. Thus, the implication Statement $1 \Rightarrow$ Statement 2 from Theorem 4.10 does not hold when $n>2$ even if $\# A=2$.

It is not difficult to check that the game form is an almost potential one; however, it cannot be represented as a perfect information game form. 
Example 4.12. Let $N=\{1,2,3\}$, player 1 choose rows, player 2 columns, and player 3 matrices:

$$
\left[\begin{array}{lll}
q & a & a \\
q & q & q \\
q & q & q
\end{array}\right]\left[\begin{array}{lll}
q & a & a \\
q & q & a \\
q & q & a
\end{array}\right] \quad\left[\begin{array}{lll}
q & a & a \\
q & a & a \\
q & q & a
\end{array}\right] \quad\left[\begin{array}{lll}
a & a & a \\
a & a & a \\
q & q & a
\end{array}\right] .
$$

Conditions (4.1) are easy to check, hence the game form is supertight. Suppose it can be represented as the fragment of a VV game form. Since the roles of $a$ and $q$ are perfectly symmetric, we may, without restricting generality, assume $q>a$; therefore, $a$ is selected when and only when $q$ is vetoed. We denote $x_{i}^{s}$ the number of black balls cast at $q$ by player $i(\in\{1,2\})$ when using $s$-th strategy, counting upwards for player 1 and from the left to right for player 2. The rightmost matrix shows that $x_{1}^{2}-x_{1}^{1}>x_{2}^{2}-x_{2}^{1}$; the leftmost, that $x_{1}^{3}-x_{1}^{2}>x_{2}^{3}-x_{2}^{2}$; therefore, $x_{1}^{3}-x_{1}^{1}>x_{2}^{3}-x_{2}^{1}$. On the other hand, each of the middle matrices shows that $x_{2}^{3}-x_{2}^{1}>x_{1}^{3}-x_{1}^{1}$.

Thus, the implication Statement $1 \Rightarrow$ Statement 3 from Theorem 4.10 does not hold when $n>2$ even if $\# A=2$. The invalidity of the converse implication when $\# A>2$ even if $n=2$ is shown by Example 4.9 .

Theorem 4.13. Every VV game form has an almost unrestricted FCP.

Proof. For every $x_{N}, y_{N} \in X_{N}, a \in A, t \in D$, and $I \in \mathcal{N}$, we define:

$$
\varepsilon\left(a, x_{N}\right)=\max \left\{\varkappa\left(a, x_{N}\right)-\lambda_{a}, 0\right\} ; \quad \mathrm{E}\left(x_{N}\right)=\sum_{a \in A} \varepsilon\left(a, x_{N}\right)
$$

(over-vetoing at $a$ and total over-vetoing);

$$
\beta\left(a, x_{N}\right)=\max \left\{\lambda_{a}-\varkappa\left(a, x_{N}\right), 0\right\}
$$

(the number of empty slots at $a$ );

$$
\begin{gathered}
C\left(x_{N}\right)=\left\{b \in A \mid \sum_{a>b} \beta\left(a, x_{N}\right)<2 \& \varepsilon\left(b, x_{N}\right)=0\right\} \ni g\left(x_{N}\right) ; \\
B\left(x_{N}\right)=x_{N}^{-1}\left(C\left(x_{N}\right)\right)
\end{gathered}
$$

(the sets of outcomes that are "close enough" to being selected and of the balls cast there);

$$
\begin{gathered}
\delta\left(x_{N}, y_{N} ; t\right)= \begin{cases}0, & \text { if } y_{N}(t)=x_{N}(t) \\
+\infty, & \text { if } y_{N}(t) \neq x_{N}(t) \& \varepsilon\left(y_{N}(t), y_{N}\right)>0 \\
d\left(y_{N}(t), x_{N}(t)\right)+1, & \text { otherwise [with } d \text { defined by }(3.5)]\end{cases} \\
\qquad \delta_{x_{-I}}\left(x_{I}, y_{I}\right)=\sum_{t \in D_{I}} \delta\left(x_{N},\left(y_{I}, x_{-I}\right) ; t\right) .
\end{gathered}
$$

For every $I \in \mathcal{N}$, the family of "costs" defined in (4.5) generates an admissible change relation $\vdash_{I}$, hence an admissible improvement relation $\bowtie$ in every derivative game. The notion of admissibility combines the ideas from the proofs of Theorems 3.7 and 3.14: the 
players shift as few balls as possible and move each of them as short a distance as possible; first of all, however, they avoid over-vetoing at any cost.

Given a list of utilities $\left\langle v_{i}\right\rangle_{i \in N}$, we define

$$
\begin{gathered}
P\left(x_{N}\right)=-\sum_{t \in B\left(x_{N}\right)} v_{\nu(t)}\left(x_{N}(t)\right) ; \\
y_{N} \succ x_{N} \rightleftharpoons\left[\forall a \in A\left[\varepsilon\left(a, x_{N}\right) \geq \varepsilon\left(a, y_{N}\right)\right] \&\right. \\
\left(\mathrm{E}\left(x_{N}\right)>\mathrm{E}\left(y_{N}\right) \text { or } B\left(x_{N}\right) \subset B\left(y_{N}\right)\right. \text { or } \\
\left.\left.\left[B\left(x_{N}\right)=B\left(y_{N}\right) \& P\left(x_{N}\right)<P\left(y_{N}\right)\right]\right)\right] .
\end{gathered}
$$

The rest of the proof consists in showing that $\succ$ is an order potential for the admissible improvement relation $\bowtie$. It seems worthwhile to describe it informally: we monitor the over-vetoing at each outcome, what balls are cast at $C\left(x_{N}\right)$ and who cast them; one strategy profile "dominates" another if either there is a strict decrease in over-vetoing, or more balls come under observation, or the balls are the same but the players try to veto worse (for them) outcomes.

Let $x_{N}, y_{N} \in X_{N}, I \in \mathcal{N}, y_{-I}=x_{-I}, a=g\left(x_{N}\right), b=g\left(y_{N}\right)$, and $D^{*}=\left\{t \in D \mid x_{N}(t) \neq\right.$ $\left.y_{N}(t)\right\}$. We have to show that $y_{N} \succ x_{N}$ in every derivative game where $y_{N} \gg_{I} x_{N}$.

By the definition of $g$, two things are necessary and sufficient for $b$ to be selected at $y_{N}$ : $b$ must not be vetoed; everything above $b$ must be vetoed. Formally:

$$
\begin{gathered}
\beta\left(b, y_{N}\right)>0=\varepsilon\left(b, y_{N}\right) ; \\
\forall c>b\left[\beta\left(c, y_{N}\right)=0\right] .
\end{gathered}
$$

We start with the demonstration of the possibility to switch from $a$ to $b$ without any increase in over-vetoing. If $\beta\left(b, x_{N}\right)=0$, then $\varepsilon\left(b, x_{N}\right)+1$ balls must be taken from $b$ to ensure (4.7a); Inequality (4.4) ensures that the total number of empty slots, $\sum_{c \in A} \beta\left(c, x_{N}\right)$, is no less than that. Therefore, the members of $I$ can make (4.7a) fulfilled without creating over-vetoing. (Since coalition $I$ was able to make $b$ selected, $\# x_{I}^{-1}(b)$ must be large enough.) Similarly, no over-vetoing could help ensuring (4.7b). Since $y_{I}$ is the least cost way to switch from $a$ to $b$, there must be $\delta_{x_{-I}}\left(x_{I}, y_{I}\right)<+\infty$. Therefore, there was no increase in overvetoing at any outcome $c \in A$ when $x_{N}$ was replaced with $y_{N}$, i.e.

$$
\forall c \in A\left[\varepsilon\left(c, y_{N}\right) \leq \varepsilon\left(c, x_{N}\right)\right]
$$

Secondly, if $\varepsilon\left(b, x_{N}\right)>0$, we have $\mathrm{E}\left(y_{N}\right)<\mathrm{E}\left(x_{N}\right)$, hence $y_{N} \succ x_{N}$ and we are home. The same conclusion is reached if $\varepsilon\left(c, y_{N}\right)<\varepsilon\left(c, x_{N}\right)$ for any $c \in A$. In the following, we assume that

$$
\begin{gathered}
\varepsilon\left(b, x_{N}\right)=0 ; \\
\forall c \in A\left[\varepsilon\left(c, y_{N}\right)=\varepsilon\left(c, x_{N}\right)\right] .
\end{gathered}
$$

Thirdly, (4.7) immediately implies $b \in C\left(y_{N}\right)$. The final argument depends on whether $b>a$ or $a>b$. 
A. Let $b>a$. By (4.9a), shifting just one ball from $b$ is enough to get that outcome selected; therefore, $D^{*}=\left\{t^{*}\right\}$ and $x_{N}\left(t^{*}\right)=b$. The minimality of $\delta\left(x_{N}, y_{N} ; t^{*}\right)$ implies that the ball goes to the nearest empty slot, which is $a$, i.e., $y_{N}\left(t^{*}\right)=a$. It is clear now that $B\left(y_{N}\right)=B\left(x_{N}\right)$ and $P\left(y_{N}\right)-P\left(x_{N}\right)=v_{\nu\left(t^{*}\right)}(b)-v_{\nu\left(t^{*}\right)}(a)>0$. Therefore, $y_{N} \succ x_{N}$ by the last disjunctive term in (4.6).

B. Let $b<a$. We again consider two alternatives. If $b \notin C\left(x_{N}\right)$, then every $c \in C\left(x_{N}\right)$ is above $b$, hence $C\left(x_{N}\right) \subset C\left(y_{N}\right)$, hence $B\left(x_{N}\right) \subset B\left(y_{N}\right)$ because at least one ball was added at $a \in C\left(y_{N}\right)$. Therefore, $y_{N} \succ x_{N}$ by the second disjunctive term in (4.6).

Now let $b \in C\left(x_{N}\right)$; then $\beta\left(a, x_{N}\right)=1$ and $\beta\left(c, x_{N}\right)=0$ for every $c$ such that $b<c<a$. Therefore, $D^{*}=\left\{t^{*}\right\}, y_{N}\left(t^{*}\right)=a$, and $x_{N}\left(t^{*}\right) \leq b$. If $x_{N}\left(t^{*}\right)=b$, then $B\left(y_{N}\right)=B\left(x_{N}\right)$ and $P\left(y_{N}\right)-P\left(x_{N}\right)>0$, hence $y_{N} \succ x_{N}$, exactly as in the case of $b>a$. This alternative must hold if $\beta\left(b, x_{N}\right)=0$. Finally, let $x_{N}\left(t^{*}\right)=c<b$, hence $\beta\left(b, x_{N}\right)>0$, hence $c \notin C\left(x_{N}\right)$. We see that $t^{*} \in B\left(y_{N}\right) \backslash B\left(x_{N}\right)$, hence $y_{N} \succ x_{N}$ exactly as in the previous paragraph.

Example 4.9 above shows that the adjective "almost unrestricted" in Theorem 4.13 cannot be dropped; actually, both improvements by player 1 there create over-vetoing.

In principle, the notion of voting by veto can be extended by considering other rules for the selection of a single non-vetoed outcome at every strategy profile. Assuming that (4.4) holds as an equality, the existence of a strong equilibrium can be shown for any $g$ (Moulin, 1983). Under a strict inequality, even Nash consistency is not ensured.

Example 4.14. Let $A=\{a, b, c, d\}, N=\{1,2\}$, every $\mu$ and $\lambda$ be equal to 1 . It is convenient to assume that each player just chooses an outcome, $x_{i}$, to veto. Let $g$ be defined as follows: If $\left\{x_{1}, x_{2}\right\} \subseteq\{c, d\}$, then $g\left(x_{N}\right)=a$ if $x_{1}=x_{2}$ and $g\left(x_{N}\right)=b$ if $x_{1} \neq x_{2}$; otherwise, $g\left(x_{N}\right)=d$ if $d$ is not vetoed whereas $g\left(x_{N}\right)=c$ if $d$ is vetoed. The rule produces this matrix:

$$
\begin{array}{llll}
d & d & d & c \\
d & d & d & c \\
d & d & a & b \\
c & c & b & a
\end{array} .
$$

Player 1 cannot ensure $g\left(x_{N}\right) \in\{a, c\}$; player 2 cannot ensure $g\left(x_{N}\right) \in\{b, d\}$. Therefore, the game form is not Nash consistent (Gurvich, 1975, 1988; Moulin, 1976).

It remains unclear to what extent Theorem 4.13 could be generalized to other mappings $g$. So far, an almost unrestricted FCP was only established for a rather peculiar tie-breaking procedure suggested by Gol'berg and Gurvich (1986), which is only defined when (4.4) holds as an equality. Example 2.1d is generated by that procedure.

\section{Acknowledgments}

Financial support from a Presidential Grant for the State Support of the Leading Scientific Schools (NSh-2982.2008.1), the Russian Foundation for Basic Research (project 08-07- 
00158), the Russian Foundation for Humanities (project 08-02-00347), and the Spanish Ministry of Education and Science (project SEJ 2007-67135) is acknowledged. I have benefitted from discussions with Vladimir Gurvich, Herve Moulin, and Endre Boros, especially during my visit to U.S. universities in 2008. I thank Herve Moulin and Francisco Marhuenda, whose effort and generosity made the trip possible. Finally, I thank two anonymous referees for the International Journal of Game Theory, who carefully read two previous versions and suggested several improvements and corrections.

\section{References}

Abdou, J., 1995. Nash and strongly consistent two-player game forms. International Journal of Game Theory 24, 345-356.

Abdou, J., 1998. Tight and effectively rectangular game forms: A Nash solvable class. Games and Economic Behavior 23, 1-11.

Abdou, J., and H. Keiding, 2003. On necessary and sufficient conditions for solvability of game forms. Mathematical Social Sciences 46, 243-260.

Andersson, D., V. Gurvich, and T.D. Hansen, 2008. On acyclicity of games with cycles. RUTCOR Research Report RRR 18-2008. Available at http://rutcor.rutgers.edu/pub/rrr/reports2008/18_2008.pdf

Boros, E., and V. Gurvich, 2003. On Nash-solvability in pure stationary strategies of finite games with perfect information which may have cycles. Mathematical Social Sciences 46, 207-241.

Boros, E., V. Gurvich, K. Makino, and D. Papp, 2010. Acyclic, or totally tight, twoperson game forms: Characterization and main properties. Discrete Mathematics 310, 1135-1151.

Boros, E., V. Gurvich, K. Makino, and W. Shao, 2007. Nash-solvable bidirected cyclic game forms. RUTCOR Research Report RRR 30-2007. Available at http://rutcor.rutgers.edu/pub/rrr/reports2007/30_2007.ps

Boros, E., V.A. Gurvich, I.E. Zverovich, and W. Shao, 2009. Assignability of 3dimensional totally tight matrices. RUTCOR Research Report RRR 02-2009. Available at http://rutcor.rutgers.edu/pub/rrr/reports2009/02_2009.pdf

Friedman, J.W., and C. Mezzetti, 2001. Learning in games by random sampling. Journal of Economic Theory 98, 55-84.

Germeier, Yu.B., and I.A. Vatel', 1974. On games with a hierarchical vector of interests. Izvestiya Akademii Nauk SSSR, Tekhnicheskaya Kibernetika, 3, 54-69 (in Russian; English translation in Engineering Cybernetics, V.12).

Gol'berg, A.I., and V.A. Gurvich, 1986. Collective choice based on the veto principle. VINITI Manuscript \#3182-V86 (in Russian).

Gurvich, V.A., 1975. Solvability of extensive games in pure strategies, Zhurnal Vychislitel'noi matematiki i matematicheskoi fiziki 15(2), 358-371 (in Russian; English translation in USSR Computational Mathematics and Mathematical Physics 15(2), 74-87). 
Gurvich, V.A., 1988. Equilibrium in pure strategies. Doklady Akademii Nauk SSSR 303, 789-793 (in Russian; English translation in Soviet Mathematics. Doklady 38, 597-602).

Holzman, R., and N. Law-yone, 1997. Strong equilibrium in congestion games. Games and Economic Behavior 21, 85-101.

Kalai, E. and D. Schmeidler, 1977. An admissible set occurring in various bargaining situations. Journal of Economic Theory 14, 402-411.

Kandori, M., and R. Rob, 1995. Evolution of equilibria in the long run: A general theory and applications. Journal of Economic Theory 65, 383-414.

Konishi, H., M. Le Breton, and S. Weber, 1997. Equilibria in a model with partial rivalry. Journal of Economic Theory 72, 225-237.

Kreps, D.M., 1990. A Course in Microeconomic Theory. Princeton University Press, Princeton.

Kukushkin, N.S., 1995. Two-person game forms guaranteeing the stability against commitment and delaying tactics. International Journal of Game Theory 24, 37-48.

Kukushkin, N.S., 1999. Potential games: A purely ordinal approach. Economics Letters 64, 279-283.

Kukushkin, N.S., 2002a. Perfect information and potential games. Games and Economic Behavior 38, 306-317.

Kukushkin, N.S., 2002b. Unfocussed Nash Equilibria. Russian Academy of Sciences, Dorodnicyn Computing Center, Moscow. A revised version available at http://www.ccas.ru/mmes/mmeda/ququ/Unfocussed.pdf

Kukushkin, N.S., 2004. Best response dynamics in finite games with additive aggregation. Games and Economic Behavior 48, 94-110.

Kukushkin, N.S., 2007a. Shapley's "2 by 2" theorem for game forms. Economics Bulletin 3, No. 33, 1-5. Available at

http://www.accessecon.com/pubs/EB/2007/Volume3/EB-07C70017A.pdf; with a typo corrected, at http://www.ccas.ru/mmes/mmeda/ququ/Shapley.pdf

Kukushkin, N.S., 2007b. Congestion games revisited. International Journal of Game Theory 36, 57-83.

Kukushkin, N.S., 2007c. Aggregation and Acyclicity in Strategic Games. Russian Academy of Sciences, Dorodnicyn Computing Center, Moscow. A version available at http://www.ccas.ru/mmes/mmeda/ququ/AgrAcl.pdf

Mariotti, M., 2000. Maximum games, dominance solvability, and coordination. Games and Economic Behavior 31, 97-105.

Milchtaich, I., 1996. Congestion games with player-specific payoff functions. Games and Economic Behavior 13, 111-124.

Milgrom, P., and J. Roberts, 1991. Adaptive and sophisticated learning in normal form games. Games and Economic Behavior 3, 82-100.

Monderer, D., and L.S. Shapley, 1996. Potential games. Games and Economic Behavior 14, 124-143. 
Moulin, H., 1976. Prolongements des jeux à deux joueurs de somme nulle. Bulletin de la Société Mathématique de France, Supplémentaire Mémoire No. 45.

Moulin, H., 1980. On strategy-proofness and single-peakedness. Public Choice 35, 437455 .

Moulin, H., 1983. The Strategy of Social Choice. North-Holland Publ., Amsterdam.

Moulin, H., 1984. Dominance solvability and Cournot stability. Mathematical Social Sciences 7, 83-102.

Mueller, D.C., 1978. Voting by veto. Journal of Public Economics 10, 57-75.

Peleg, B., 1978. Consistent voting systems. Econometrica 46, 153-161.

Rosenthal, R.W., 1973. A class of games possessing pure-strategy Nash equilibria. International Journal of Game Theory 2, 65-67.

Sela, A., 1992. Learning Processes in Games. M. Sc. Thesis. The Technion, Haifa (in Hebrew).

Topkis, D.M., 1979. Equilibrium points in nonzero-sum n-person submodular games. SIAM Journal on Control and Optimization 17, 773-787.

Vives, X., 1990. Nash equilibrium with strategic complementarities. Journal of Mathematical Economics 19, 305-321.

Voorneveld, M., and H. Norde, 1996. A characterization of ordinal potential games. Games and Economic Behavior 19, 235-242.

Young, H.P., 1993. The evolution of conventions. Econometrica 61, 57-84. 